\author{
N. Arnold, S. Baydoun, L. Chalak \& Th. Raus
}

\title{
A contribution to the flora and ethnobotanical knowledge of Mount Hermon, Lebanon
}

\begin{abstract}
Arnold, N., Baydoun, S., Chalak, L. \& Raus, Th.: A contribution to the flora and ethnobotanical knowledge of Mount Hermon, Lebanon. - Fl. Medit. 25: 13-55. 2015. — ISSN: 1120-4052 printed, 2240-4538 online.

Despite the generally recognized role of Mt Hermon as a key area of biodiversity, there is still a lack of sufficient data on the vascular flora of the Lebanese side of the mountain. To fill this gap a list of 221 phanerogams, collected during 2011-2014 at altitudes of 1100 to $2800 \mathrm{~m}$, is presented including information on the eco-geographical traits of the taxa. Ethnobotanical knowledge of identified species was gathered through interviews and focus group meetings with herbalists, folk medicine healers, bee keepers and knowledgeable elderly people of the local Lebanese community. Among the 53 ascertained plant families Asteraceae (32 spp.), Lamiaceae (25 spp.), Apiaceae (17 spp.), Fabaceae (14 spp.) and Rosaceae (14 spp.) form the major shares. Among the observed 221 taxa, 24 endemics (Lebanon and Syria) are recorded and 115 taxa are for the first time detected in the mountain ecosystem. Associated with the valuable wealth of traditional ethnobotanical knowledge gathered, these findings can highlight the importance of Mt Hermon in terms of flora diversity and its valuable potential in enhancing the livelihood of local human communities. Thus, it is of significance to set the necessary approach to include the mountaine among the high priority areas considered for the national and global biodiversity conservation efforts.
\end{abstract}

Key words: Ethnobotany, Floristics, Plant use, Middle East.

\section{Introduction}

Situated on the E coast of the Mediterranean, Lebanon is considered one of the important centers of plant biodiversity and endemism (Médial \& Quézel 1977, 1999; CEPF 2010). Despite the small size of the country (i.e. of $10.452 \mathrm{~km}^{2}$ only), sharp contrasts in climatic and geomorphological conditions have resulted in seven different phyto-association zones. The $4^{\text {th }}$ Lebanon national report on Conventional Biological Diversity (MoE \& al. 2009, 2011) noted that the country is home to over 2,600 terrestrial plant species with a high rate $(12 \%)$ of endemism including $8.5 \%$ as broad endemics (Lebanon, Syria, and Palestine) and 3.5\% as narrow endemics to Lebanon. The country is recognized to support exceptionally high numbers of rare and threatened species, most of which are reported to 
inhabit high mountain summits, namely Mt Makmel, Mt Sannine, Qamouaa, Ehden, and Mt Hermon. The isolation effect characterizing these mountain peaks renders them as a reservoir for endemic species (MoE \& al.2009; CEPF 2010).

Mt Hermon, a mountain ridge in the Anti-Lebanon range, extends for $35 \mathrm{~km}$ in a NESW direction and rises from 300 to $2814 \mathrm{~m}$. The mountain climate is of a Mediterranean type with rainy, cold winters and hot, dry summers. The parent rock material consists of homogeneous, hard Jurassic limestone, dolomites and occasional veins of basalt which are broken by faults and solution channels to form a karst topography. Quaternary deposits formed of gravels, sands and clays overlie the older formations in some areas. The local geomorphology is characterized by steep slopes and rocky sharp clefts with pronounced active weathering processes at higher altitudes resulting from snow coverage of all peaks for most of the year. As most soils in Lebanon the soil of Mt Hermon is characterized by fragility, poor consistency and shallowness especially on sloping terrains. Several studies of the E Mediterranean ecosystems indicate that the vegetation cover of Mt Hermon was prior to human intervention composed of a climax community of oak woodland and plenty of shrubs and deciduous trees accompanied by a rich herbaceous vegetation (Abou Zakhem1989; Danin 1992; Auerbach \& Shmida 1993). Three main vegetation belts have been distinguished in the mountain (Zohary 1973): Evergreen Mediterranean Maquis (3001300 m), Xero-Montane Open Forest (1300-1900 m) and Subalpine Mountain Steppe or Tragacanth Belt (1900-2800 m).

In spite of the insufficient studies and awareness of the vital ecological services Mt Hermon provides, preliminary data indicate the importance of the mountain as a source for subsistence for a population of around 60.000 people of the local vulnerable communities in the towns of Rachaiya, Hasbaya, Yanta, Aaiha, Kfer Qouq, Kfer Chouba, Chebaa, Ain Aata, and Beit Lahia. The mountain offers a wide range of ecosystem products mainly in terms of fresh water and agricultural crops. Moreover, important cultural, recreational, and spiritual sources are offered. Grapes, olives, honey, grape molasses, wine and vinegar, traditional dairy products, and medicinal plants form some of the most traditionally key products. Goat grazing also stands as one of the important life-support systems of the mountain ecosystem that highly influences both vegetations and locals. Hermon community is endowed with a rich traditional ethnobotanical knowledge system on folk medicine resulting from an extensive prosperous history of interaction with the flora of the mountain (Malychef 1989; Apostolides-Arnold 1991; El Beyrouthy \& Arnold 2008; Abou Chaar 2004; El Beyrouthy \& al. 2008). In addition to these traditional innumerable interactions with its livelihood, the mountain held great religious significance for many of the ancient human settlements and civilizations. In regional Christian tradition, Mt Hermon is recognized as the site of the transfiguration of Jesus, annually celebrated by the mount community and religious tourists from Lebanon and countries across the world on August $6^{\text {th }}$. Hundreds of people participate in a seven-hour march from Rachaiya to the Hermon summit which Jesus Christ is believed to have visited thousands of years ago. This spiritual value has also been a contributor to the social and economic development of the local harmonious blend of religious groups of Christians, Sunni Muslims, Shiaa Muslims and the largest settlement of Druze in Lebanon.

Although the flora of the NW side of Mt Hermon in Lebanon has not been subject to any comprehensive assessments, the mountain is considered as one of the Important Plant 
Areas of the E Mediterranean Region. It is indicated to have a high rate of endemism and host many rare plant species (Yazbek \& al. 2011). More than 100 taxa endemic to Mt Hermon and the Anti-Lebanon Range were noted by Medial \& Quezel (1997).

However, as most of the ecosystems in the country the natural wealth of Mt Hermon has been subject to various human and natural pressures highly impacting its plants and all other types of wildlife. Losing important habitats and species, over-exploitation of resources, grazing, invasive alien species, urban expansion, and increasing impact of climate change are among the challenges facing the mountain and its surrounding community.

This study presents a contribution of 221 taxa to a forthcoming checklist of the vascular plants of Mount Hermon in Lebanon along with the traditional ethnobotanical knowledge gathered from the ancient interactions between the mountain and its livelihood. The list is the result of original, hitherto unpublished field surveys, partially guided and supplemented by information from Mouterde (1966, 1970, 1983), Davis (1965-1985), Greuter $\&$ al. $(1984,1986,1989)$ and Zohary $(1966,1970)$. It aims at providing a basic knowledge to programs on national and regional biodiversity conservation and sustainable management of natural resources. It also aims at contributing to efforts to maintain the life-support systems provided by Mt Hermon's ecosystem and ensure greater resilience of local human communities.

\section{Materials and methods}

A floristic field survey along the elevation gradient of the Lebanese side of Mt Hermon was launched in May 2011 and is still continuing. As indicated on the map (Fig. 1), the sites visited were distributed between 1100 and $2420 \mathrm{~m}$ of altitude covering the main accessible hills and valleys such as Rachaiya (1100 m), Chrabbé (1226 m), Berket El Yabsé (1234 m), Ouastani (1264 m), Dalayel (1310 m), Shâm El-Jabal (1325 m), Manara (1450 m), Hima Hassan (1465 m), Aarqoub (1624 m), Wadi Al-Waseea (1715 m), Beit ElFransaoui (2250 m), Journaya $(2410 \mathrm{~m})$ and Chaghour Ed-Dahab (2814 m). These sites were subject to a preliminary characterization of geomorphology and habitat, i.e. topography, type of soil and rocks, and type of vegetation. Plant specimens were collected at random during flowering or fructification stage. The specimens were immediately pressed and accompanied by comprehensive notes of GPS coordinates, altitude, plant description as well as specific details of micro-habitat. Images of the investigated taxa were also retained. Mounted specimens are stored in the herbarium of the Research Center for Environment and Development, Beirut Arab University, Bekaa.

Plant identification was performed using the determination keys of Mouterde (1966, 1970, 1983). Nomenclature and plant family delimitation follows Euro+Med (2006-) as far as published, and Greuter \& al. (1984, 1986, 1989), with Compositae (Greuter 2008) adapted to Asteraceae, and family classification of APG III (Angiosperm Phylogeny Group 2009) given if deviating.

An ethnobotanical survey of the identified plant taxa was undertaken to collect the breadth of popular inherited knowledge from traditional herbalists, folk healers and elderly knowledgeable villagers. Data was collected through focus groups, semi-structured questionnaire (Table 1) and personal free listing interviews during field trips to the studied sites. 


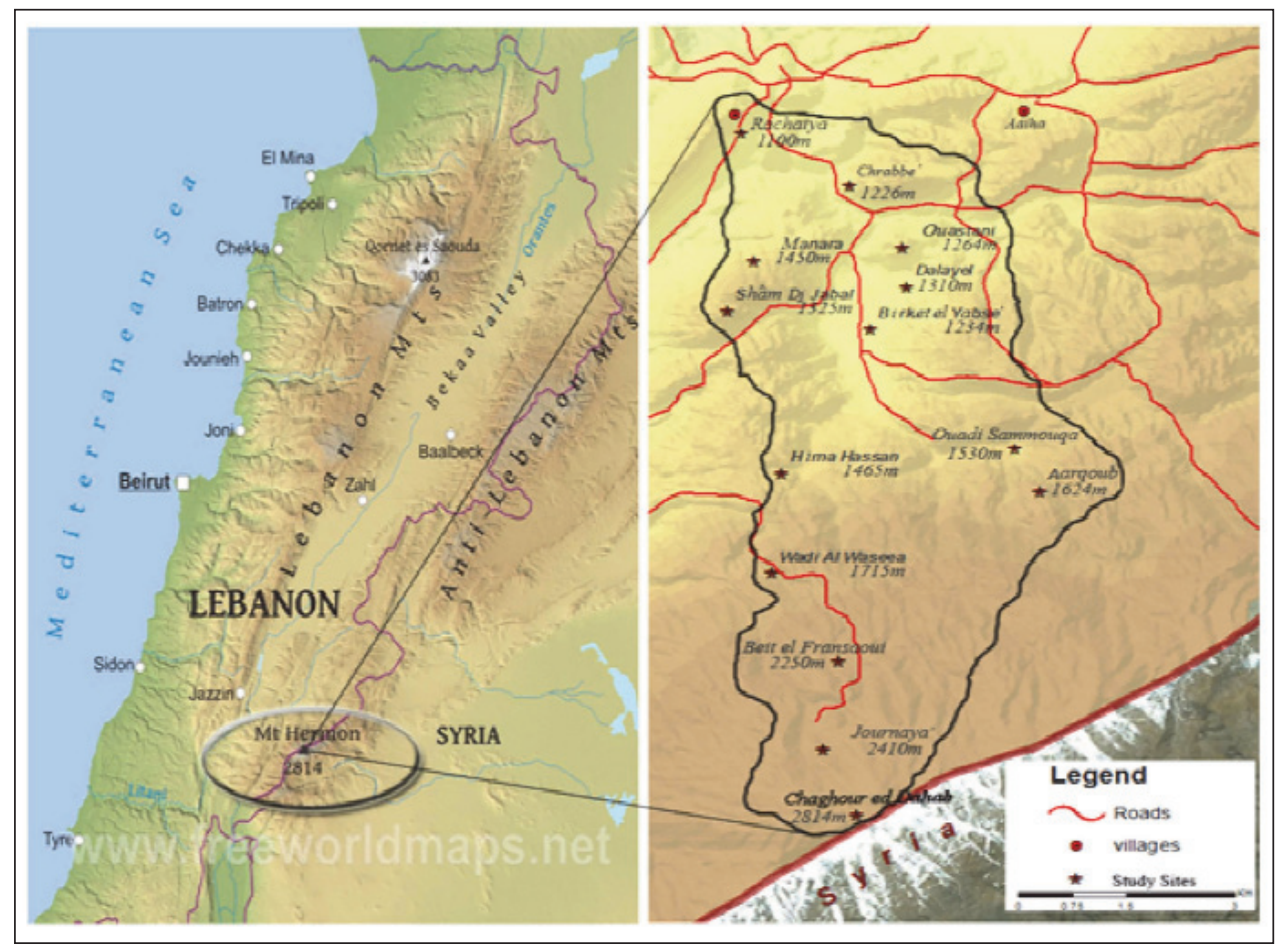

Fig. 1. Hermon Mountain: study area and sites visited. - Source: www.freeworldmaps.net and adaption of Google Earth.

Interviews were mainly focused on knowledge about the past and present use, mode of preparation, parts of the plants used, way of administration, and types of diseases treated.

\section{Results}

\section{Taxonomic allocation of the vouchers collected}

A numberof 221 species was, to date, collected and identified in this project. The species belong to 53 families and 165 genera, including one species of Pteridophyta and 220 species of Spermatophyta (Table 2). The rate of endemics to Lebanon and Syria amounts to 24 taxa (10.9\%). Excluded from the list are the cultivated varieties of olives, tomatoes, chick peas, vines and wheat.

\section{Ethnobotanical traditional knowledge documented}

The survey showed that Mt Hermon's flora has a large number of medicinal plants (169 taxa) to treat a wide spectrum of human ailments. Many people are still depending on medicinal plants, at least for the treatment of some simple diseases as cold, cough, fever, 
Table 1. Semi-structured survey questionnaire used for gathering the ethobotanical knowledge on the Mt Hermon flora.
Name:
Gender and Age:
Profession/Education:
Date / Collector:

1. Do you have any knowledge of the benefits, uses and toxicity of plants?

2. What is the vernacular Arabic name of the plant (collected plant or voucher specimen and photo)?

3. What are the main uses of the plant?
a. Food
f. Natural products (essential oil, oleoresin)
b. Medicinal (human /animal)
g. Timber/fuel
c. Flavor and aroma
h. Melliferous
d. Ornamental
i. Cultural, religious, social
e. Animal fodder
j. Others

4. How long has the use of the plant been known to you?

5. What is the source of your information?
a. Oral transmission from experienced old people
b. Inherited manuscript
c. Others

6. How frequently do you use the plant?

7. If edible

a. Whichpart is eaten?

b. How (raw or cooked)?

8. If medicinal
a. Whicht diseases are treated by the plant?
b. Which part is used?
c. How is it prepared and adminstrated? (infusion, decoction, crushed, maceration ...etc.)

9. If other uses,
a. Which part?
b. Describe how.

10. Is the plant collected from the wild and sold?

11. Is the plant cultivated? For which reason?

Table 2. Taxonomic allocation of collected vouchers from Mount Hermon, Lebanon.

\begin{tabular}{|l|c|c|c|}
\hline \multicolumn{1}{|c|}{ Plant Group } & Families & Genera & Species \\
\hline Pteridophyta & 1 & 1 & 1 \\
\hline Gymnospermae & 2 & 2 & 2 \\
\hline Angiospermae Dicotyledoneae & 40 & 139 & 187 \\
\hline Angiospermae Monocotyledoneae & 10 & 23 & 31 \\
\hline Total & 53 & 165 & 221 \\
\hline
\end{tabular}


headache, poison bites, skin diseases and tooth infections. It is necessary to acquire and preserve this traditional system of medicine by proper documentation. This traditional knowledge on the indigenous uses of the medicinal plants could boost innovations in the pharmaceutical industry and have many beneficial applications such as new medicinal trails for some diseases like jaundice, cancer, diabetes and rheumatism of which the health care sector in Lebanon will further develop.

\section{Annotated floristic catalogue}

In the list of 221 collected species presented here, families, genera and species are arranged alphabetically. Accepted names are set in bold-face italics; they are referred to the recent basic floras and checklists concerning the area (Euro+Med 2006-; Greuter \& al. 1984, 1986, 1989, 2008; Mouterde 1966, 1970, 1983), and to selected taxonomic literature. Synonyms are limited to names adopted in these reference works. Scientific names are followed by vernacular names in English and Arabic in current regional Lebanese use (Nehmé 2008), life-form, habit and height of growth, flowering time, eco-geographical details both locally (Mt Hermon voucher) and country-wide (Lebanon), habitat traits, and the ethnobotanical usage as recorded during this study.

Abbreviations used: Ar: Arabic; En: English; Ft: Flowering time; Hab: Habitat; Lf: Lifeform; Syn: Synonymy.

\section{Acanthaceae}

Acanthus syriacus Boiss. - En: Syrian Acanthus, Syrian Bear's Breech. - Ar: Shawk El Jamal, Kaf El Deb. - Lf: Perennial shrub or herb, spiny, 40-80 cm. - Ft: May-July. - Mt Hermon: 1445 m, $33^{\circ} 28^{\prime} 29^{\prime}$ N, 35 51'06”E. - Lebanon: Sarafand, Saida, Beirut, Nahr El Kalb, Tripoli, Aabey, Moukhtara, Jamhour, Terbol, Dahr Al Baidar, Sannine, Rachaiya. - Hab: Meadows, roadsides, margins of cultivated fields, red clay soil (terra rossa). - Uses: Infusion of fruits and leaves, leaves extract for wound healing, emollient, anti-inflammatory.

\section{Aceraceae (Sapindaceae)}

Acer monspessulanum subsp. microphyllum (Boiss.) Bornm. - Syn: A. microphyllum (Boiss.) Dinsm. non Opiz, A. hermoneum (Bornm.) Bornm. \& Schwer., A. syriacum Boiss. \& Gaill. var. hermoneum Bornm.; Lectotype: Mt Hermon, Kotschy 240 (Gelderen \& al. 1994:185). Confirmation of the Hermon population of this taxon which was queried by Gelderen \& al. (1.c.) to still exist. - En: Montpellier Maple. - Ar: Kaykab Haramon, Kaykab Jabal El Sheikh. - Lf: Shrub or small tree, 100-

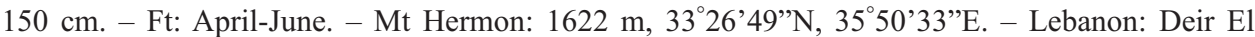
Ahmar, Ainata, Litani towards Yohmor, Ouadi El Karm, Chebaa and Rachaiya. - Hab: Woodlands, limestone rocks, sunny places. - Uses: Decoction of seeds is used for cough.

\section{Amaryllidaceae (Alliaceae, Liliaceae s. 1.)}

Allium ampeloprasum L. - En: Wild Leek, Broadleaf Wild Leek. - Ar: Koraat Barri Ahmar. - Lf: Perennial herb, bulbous geophyte, $150 \mathrm{~cm}$. - Ft: April-June. - Mt Hermon: 1292 m, 33²8’55’N, 35 51'73’E. - Lebanon: Nahr El Kalb, Aabey, Jamhour, Aintoura, Baskinta, Bcharre, Falougha, 
Zahle, Hasbani. - Hab: Limestone fields, damp meadows, roadsides, sunny places. - Uses: Bulbs are used as antiseptic and for intestinal worms as anthelminthic. Plant juice is also an insect repellent.

Allium chloranthum Boiss. - En: Mountain garlic. - Ar: Thum Mukhdarr Al Zahr. - Lf: Perennial herb, bulbous geophyte, 30-100 cm. - Ft: August-September. - Mt Hermon: 1219 m, 33 30'00”N, 35 50'44”'E. - Lebanon: Ariz, Ehden. - Hab: Rocky mountain slopes, roadsides, low mountain ranges, sunny places. - Uses: Edible and ornamental plant.

Allium rotundum L. - Syn: A. cilicium Boiss. - En: Sand Leek. - Ar: Thoom Ahmar. - Lf: Perennial herb, bulbous geophyte, 30-80 cm. - Ft: April-May. - Mt Hermon: 1191 m, 3328'55’N, 35 51'73”E. - Lebanon: Aabey, Douma, Jabal El Barouk, Ehmej, Ain Zhalta, Hasroun, Bcharre, Sannine, Mairouba, Dahr Al Baidar, Rachaiya, Zahle, Rayak. - Hab: Limestone fields, damp meadows, roadsides, sunny places. - Uses: Crushed fresh bulbs mixed with honey is applied locally as cataplasm to soothe pains and avoid suppuration. Bulbs are used as diuretic, vermifuge and hypoglycemic.

\section{Anacardiaceae}

Pistacia terebinthus subsp. palaestina (Boiss.) Engl. - Syn: P. palaestina Boiss. - En: Terebinth Tree, Turpentine Tree. - Ar: Botom. - Lf: Tree, 7-8 m. - Ft: February-April. - Mt Hermon: 1373 m, $33^{\circ} 27^{\prime} 38^{\prime \prime}$, 35 50'59’'E. - Lebanon: Ras El Biyada, Saida, Beirut, Nahr El Kalb, Ras Chekka, Deir Al Qamar, Beit Meri, Wadi Qannoubine, Ehden. - Hab: Woodlands, rocky areas in high mountain ranges. - Uses: Infusion of leaves is used as diuretic.

Rhus coriaria L. - En: Elm-leaved Sumach, Tanner's Sumach, Sicilian Sumac, Sumac. - Ar: Summaq. - Lf: Shrub, 1-3 m. - Ft: April-June. - Mt Hermon: 1373 m, 33²7’38’N, 3550'59’E. Lebanon: Beirut, Tripoli, Jamhour, Ain Zhalta, Douma, Ghazir, Barouk, Aabey, Ijdabra above Batroun. - Hab: Near cultivated areas, dry hillsides. - Uses: Tannins are found in all parts of the plant, particularly bark and roots, are used in tanning leather. Fruits are powdered and used as a spice in Lebanese cooking to add a sour bitter taste to salads, grilled meat or chicken. Seed powder is also used as antidiarrhoeic and astringent.

\section{Apiaceae (Umbelliferae)}

Artedia squamata L. - En: Crown Flower, Fennel-leaved Flower. - Ar: Artedeyya Horshofeyya. Lf: Annual herb, 20-50 cm. - Ft: March-May. - Mt Hermon : 1353 m, 33²7’43”N, 35 51'03”E. Lebanon: Saida, Beirut, Tripoli, Jamhour, Baabda, Bsarma, Koura. - Hab: Gravel soil, open shrubby places, roadsides, low mountain ranges, dry and sunny places. - Uses: Infusion of seeds is used as stomachic.

Conium maculatum L. - En:Spotted Hemlock. - Ar: Shawkaran Mubaqqa. - Lf: Biennial herb, 12 m. - Ft: April-June. - Mt Hermon: 1197 m, 3329’47’N, 3550’35”E. - Lebanon: Beirut, Tripoli, Ehden, Ariz, Taanayel, Zahle, Bekaa, Ouadi el Harir. - Hab: fields, abandoned lands, roadsides, low mountain ranges, sunny places rich in nitrogen. - Uses: Toxic (all parts) and medicinal plant. Used for respiratory system diseases, joint pains and cramps, anxiety, skin and bladder infections.

Daucus carota subsp. maximus (Desf.) Ball - Syn: D. blanchei Reut. - En: Wild Carrot, Bird's Nest, Bishop's Lace, Queen Anne's Lace. - Ar: Jazar Barri Kabeer. - Lf: Biennial herb, 1-2 m. - Ft: April-July. - Mt Hermon: 1292 m, 33²8'54’N, 3551'35'E. - Lebanon: Beirut, Nahr El Kalb, 
Chekka, Aabey, Tripoli, Baabda, Ain Bsaba, Mrouj, Choueir, Roumie, Mar Youhanna Maroun, Qraiye, Bcharre, Falougha, Qornayel, Rachaiya. - Hab: Rough grassy grounds, roadsides, hillslopes, stony pastures, cultivated lands, low mountain ranges, sunny places. - Uses: Fruit is diuretic and anti-inflammatory for hemorrhoids in urinary and uterine infections, recommended to disintegrate urinary calculi and kidney stones, whole plant is antispasmodic and laxative.

Dichoropetalum junceum (Boiss.) Pimenov \& Kljuykov - Syn: Peucedanum junceum (Boiss.) Mouterde, P. spreitzenhoferi Dingler, Johrenia juncea Boiss. - En: Sulphur-wort. - Ar: Posedian Shabeeh Asal. - Lf: Perennial herb, 100-200 cm. - Ft: June-September. - Mt Hermon: 2305 m, $33^{\circ} 25^{\prime} 39$ 'N, 35 50’31'E. - Lebanon: Qraiye, Feytroun, Berdawni, Kamed El Laouz, Rachaiya. Hab: Calcareous rocky slopes, crevices, dry and sunny places. - Uses: Decoction of fresh roots or crushed roots past with salt is freshly administered as a digestive for cattle and sheep.

Eryngium billardierei F. Delaroche - En: Labillardiere's Eryngo. - Ar: Shindab Labillardiere. - Lf: Perennial herb, 30-100 cm. - Ft: June-September. - Mt Hermon: 1409 m, 33²7’25'N, 35 50'53”E. - Lebanon: Jabal El Knisse, Ariz, Dimane, Yammoune, Jabal Sannine, Faraiya, Mt Hermon. - Hab: hillsides, cultivated fields, roadsides, dry places, red clay soil. - Uses: Medicinal plant used as antiinflamatory, against snake bites, scorpion stings, skin and oral pathogens.

Eryngium creticum Lam. - En: Field Eryngo. - Ar: Kors Anne. - Lf: Perennial herb, spiny, 30-60 cm. - Ft: May-September. - Mt Hermon: 1250 m, 33 28'54’N, 3551’35”E. - Lebanon: Tyre, Saida, Beirut, Beirut River, Broumana, Deir Karkafe, Aabey, Dimane, Taanayel, Ouadi El Harir. - Hab: Dry and damp soil, several types of soil, hillsides, roadsides, cultivated and abandoned lands, cliff crevices. - Uses: Decoction of stems is taken orally to treat cough, kidney inflammation, and diabetes. Decoction of roots is used as diuretic and to treat kidney stones, uterine ailments, and some skin diseases. The plant juice is also an antidote for scorpion stings.

Eryngium glomeratum Lam. - En: Glomerate Eryngo. - Ar: Shendab Moutajammei. - Lf: Perennial herb, spiny, 30-100 cm. - Ft: June-August. - Mt Hermon: 2345 m, 3325'35'N, 35 50'40”E. Lebanon: Saida, Beirut River, Broumana, Bikfayia, Aabey, Douma, Ariz, Ehden, Ksara, Mt Hermon area. - Hab: Dry and stony slopes, grassy grounds, meadows, vineyards, gravel soil, high mountain ranges. - Uses: Decoction of root is used diuretic to treat kidney stones, some skin diseases and for haemorrhoids.

Ferula hermonis Boiss. - En: Hermon Giant Fennel. - Ar: Shirch El Zaloue, Zallouh. - Lf: Perennial tall herb, very aromatic, 1-1.5 m. - Ft: May-August. - Mt Hermon: 2740 m, 33²5'08'N, 35 51'50'E. - Lebanon: occurrence confirmed (queried by Euro+Med 2006- based on Mouterde 1970: 640), confined to Mt Hermon area. Endemic to Lebanon and Syria. - Hab: Among the calcareous rocks, dry and rocky slopes, high mountain ranges, sunny and shady places. - Uses: Decoction of rhizome is widely used as aphrodisiac to enhance potency and sexual activity in traditional medicine, and to relieve the symptoms of treatment of fatigue. Resin of cut stem is also used in cough and asthma. The rhizomes are sold by herbalists in Lebanon and Syria, and exported mainly to Arabic Middle Eastern countries. Traditionally, the hydrolate or powdered rhizome mixed with honey of melliferous flowers is taken orally for these various purposes and decoction from roots and seeds (50 g / liter of water) is boiled and taken orally, $150 \mathrm{ml}, 2$ times/day until improvement occurs.

Ferulago frigida Boiss. - En: Mountain Ferulago. - Ar: Anijezan Jabali. - Lf: Perennial herb, 60100 cm. - Ft: June-July. - Mt Hermon: 2300 m, 3325'36”N, 35 50’28”'E. - Lebanon: Ghazir, Ehden, 
Mar Semaan, Al Danniyeh, Laqlouq, Ariz, Jabal El Knisse, Jabal El Qamouaa, Ammiq forest, Sannine summit above Ainata. Endemic to Lebanon and Syria. - Hab: Calcareous rocks in high mountain ranges. - Uses: Essential oils of aerial parts and seeds have antibacterial properties. Flowers and leaves are used as sedative, aphrodisiac and to treat digestive pains, ulcers, snake bite, intestinal worms and haemorrhoids.

Heracleum humile Sm. - En: Berce Humble. - Ar: Harakliyya Moutawadiaa. - Lf: Perennial herb, 20-60 cm. - Ft: June-August. - Mt Hermon: 1489 m, 3327'24’N, 3551'38’E. - Lebanon: Jabal Sannine, Ariz, Ain El Qarn, Mt Hermon. - Hab: Mountain rocks, calcareous plateaus at medium altitudes. - Uses: Grounded root paste is used in snake bites and to treat fever, abdominal cramps caused by intestinal worms. Traditionally, the plant is also used to treat nerve disorders. Plant is also considered as a good feed for goats to increase milk production.

Lagoecia cuminoides L. - En: Bastard Cumin. - Ar: Karawiyah Jabaliyyah. - Lf: Annual herb, 2050 cm. - Ft: March-May. - Mt Hermon: 1197 m, 33 29'47’N, 3550’35”E. - Lebanon: Tyr, Saida, Damour, Beirut, Tripoli, Beit Meri, Baalbek, Yaat. - Hab: Cultivated fields, pastures, abandoned land, roadsides, hillsides, low mountain ranges, sunny places. - Uses: Condiment (cumin substitute).

Malabaila secacul (Mill.) Boiss. - Syn: Tordylium secacul Mill., Pastinaca secacul (Mill.) Sol. En: Arabian Hart-wort. - Ar: Secacoul, Hachkal, Shakakel. - Lf: Perennial herb, 30-80 cm. - Ft: April-June. - Mt Hermon: 1292 m, 3328'55'N, 3551'73”E. - Lebanon: Ehden forests, Qozhaiya, Mdeyrej, Beit Mounzer, Towmat Jezzine, Hasroun, Jezzine, Afqa, Chtaura (Khan Mourad), Zahle, Wadi El Harir, Mt Hermon area. - Hab: Dry shrubby areas, scrubs, cultivated and abandoned lands, grassy grounds, sunny places, low mountain ranges. - Uses: Leaves and flowers are externally used in the form of poultice as analgesic to treat pains.

Pimpinella corymbosa Boiss. - En: Corymbed burnet-saxifrage. - Ar: Bembenella Aazkeyia. - Lf: perennial herb, 30-60 cm. - Ft: May-July. - Mt Hermon: 1275 m, 3328'13”N, 35 51'19'E. Lebanon: Zahle, Ksara, Baalbek, Ras Baalbek, Hermel, Rachaiya, Aiha, Ouadi El Harir. - Hab: dry and stony hillslopes, orchards, low mountain ranges. - Uses: Medicinal plant used as carminative, diuretic, antipoison and sedative. Animal feed to increase milk secretion.

Pimpinella tragium Vill. - En: Tragium Burnet Saxifrage. - Ar: Yansoon Barri. - Lf: Perennial herb, 30-50 cm. - Ft: June-July. - Mt Hermon: 2306 m, 33²5’37’N, 3550'32”E. - Lebanon: Bikfayia, Jabal El Barouk, Hasroun, Ainata, Ariz, Knisse, Jabal Sannine, Makmel, Yammoune, Deir El Ahmar. Infraspecific allocation of Lebanese populations unsettled so far but subsp. lithophila (Schischk.) Tutin seems most likely. - Hab: Calcareous rocky slopes, crevices, clayey doline bottoms, sometimes growing through spiny and dwarf shrubs, high mountain ranges. - Uses: Infusion of seeds is taken as carminative, diuretic and stomachic.

Prangos asperula Boiss. - En: Rough Prangos. - Ar: Farsh Al Dabee. - Lf: Perennial herb, 80-100 cm. - Ft: May-June. - Mt Hermon: 1197 m, altitude, 3329'47’N, 3550’35’'E. - Lebanon: Mdeyrej, Dahr Al Baidar, Khan Sannine, Jabal El Knisse, Tannourine, Ehden, Taanayel, Kaoukaba. Endemic to Lebanon and Syria. - Hab: Sunny slopes, low mountain ranges. - Uses: Medicinal used for dysmenorrhea and amenorrhoea.

Tordylium aegyptiacum (L.) Lam. - Syn: Hasselquistia aegyptiaca L. - En: Egyptian Hart-wort. Ar: Masriyya. - Lf: Annual herb, 30-40 cm. - Ft: February-April. - Mt Hermon: 1292 m, 33²8'55'N, 
35 51'37’'E. - Lebanon: Saida, Tripoli, Aabey, Tyre, Aintoura, Hasbaya (Mt Hermon region). - Hab: Dry shrubby areas, scrubs, cultivated and abandoned lands, grassy grounds, sunny places, low mountain ranges. - Uses: Plant (flowers and seeds) are used as diuretic in kidney diseases. Infusion of seeds is antifungal, anthelmintic, expectorant, and used in the treatment of pruritis (itching and scabies). Roots juice is used in the treatment of indigestion and high stomach acidity.

Torilis leptophylla (L.) Rchb. f. - Syn: Caucalis leptophylla L. - En: Fine Leaved Hedge Parsley. Ar: Bakdounes Barri. - Lf: Annual herb, 20-40 cm. - Ft: March-June. - Mt Hermon: $1250 \mathrm{~m}$, $33^{\circ} 28^{\prime} 55^{\prime}$ N, 35 51'37'E. - Lebanon: Beirut, Tripoli, Aintoura, Hasroun, Jabal Terbol, Bzemmar, Baskinta, Jabal El Knisse, Ain Zhalta, Baalbek, Ammiq, Dbaiye, Taanayel. - Hab: Cultivated and abandoned lands, meadows, roadsides, low mountain ranges. - Uses: Infusion of plants and fruits is used as intestinal antiseptic and antibacterial in gastro-intestinal illness.

\section{Apocynaceae}

Nerium oleander L. - En: Oleander. - Ar: Sam Al Himar. - Lf: Evergreen shrub, 1-4 m. - Ft: April-October. - Mt Hermon: 855 m, 3329'04”N, 3546’32”E. - Lebanon: Beirut, Nahr El Kalb, Tripoli, Damour, Baalbek. - Hab: Damp soil, river sides, roadsides, low mountain ranges. - Uses: Poisonous plant. Fresh leaves are locally applied in the treatment of skin diseases and given animals to heal ulcers.

Vinca herbacea Waldst. \&Kit. - Syn: V. libanotica Zucc. - En: Herbaceous Periwinkle. - Ar: Kodab Oushbi. - Lf: Evergreen, spreading subshrub, 30-40 cm. - Ft: March-April. - Mt Hermon: $1168 \mathrm{~m}, 33^{\circ} 30^{\prime} 02^{\prime \prime} \mathrm{N}, 35^{\circ} 49^{\prime} 51^{\prime \prime} \mathrm{E}$. - Lebanon: Marjayoun. - Hab: Open shrubby areas, hedges, rocky red clay soil. - Uses: None documented.

\section{Araceae}

Arum palaestinum Boiss. - En: Palestine Arum. - Ar: Louf Falastini. - Lf: Perennial herb, tuberous geophyte, 20-40 cm. - Ft: March-May. - Mount Hermon: 1157 m, 33³0'05'N, 3549'49”E. Lebanon: Damour, Tripoli, Ain Trez, Rechmaiya, Maifouq, Kfar Hilda, Ehmej, Jabal Terbol, Marjayoun, Hazerta, Hasbaya. - Hab: Rocky slopes in the hills and mountains, shady places. - Uses: Raw plant is toxic and very irritant. Decoction of leaves is externally used for rheumatism.

Biarum bovei Blume - Syn: Homaida bovei (Blume) Kuntze, Ischarum bovei (Blume) Schott - En: Bové's Biarum. - Ar: Moaanaka. - Lf: Tuberous geophyte, 30-40 cm. - Ft: October-December. - Mt Hermon: 2057 m, 33²6’10”N, 3550’21’E. - Lebanon: Btater, Bhamdoun, Knisse, Kefraiya, Ablah, Ksara, Yahfoufa, Rachaiya, Mt Hermon. - Hab: Among rocks in shady places, low and high mountain ranges. - Uses: Ornamental plant.

\section{Asparagaceae (Hyacinthaceae, Liliaceae s. l.)}

Asparagus acutifolius L. - En: Wild Asparagus. - Ar: Halyoun Barri. - Lf: Perennial evergreen rhizomatous shrub, 30-70 cm. - Ft: August-September. - Mt Hermon: $1292 \mathrm{~m}, 33^{\circ} 28^{\prime} 55^{\prime \prime} \mathrm{N}$, 3551'73”E. - Lebanon: Naher El Damour, Beirut River, Nahr El Kalb, Tripoli, Araya, Kahale, Bleybel, Aabey, Bikfayia, Zahle, Broumana. - Hab: Dry places, hedges, rocky and grassy grounds. - Uses: Young tender shoots in appetizers make the joy of gourmets. Decoction of pounded roots is used for headache and to treat jaundice and liver disorder. 
Drimia maritima (L.) Stearn - Syn: Urginea maritima (L.) Baker, U. scilla Steinh., Scilla maritima L. - En: Maritime Squill. - Ar: Bassal El Far. - Lf: Perennial herb, bulbous geophyte, 40-160 cm. Ft: July-October. - Mt Hermon: 1422 m, 33²8’47’N, 35 50'39’E. - Lebanon: Beirut, Nahr El Kalb, Ras Chekka, Tripoli, Kahale, Akkar, Et Tlaile. - Hab: Rocky slopes, medium mountain ranges, damp and dry places. - Uses: All plant parts cause skin irritation and allergy. Bulb is toxic to man and rodents. Fresh bulbs are used in the treatment of rheumatism, edema and gout.

Leopoldia comosa (L.) Parl. - Syn: Muscari comosum (L.) Mill. - En: Purple Grape Hyacinth. - Ar: Holhol Ankoudi. - Lf: Perennial herb, bulbous geophyte, 30-70 cm. - Ft: January-March. - Mt Hermon: 1290 m, 33²8'53'N, 35 51'47'E. - Lebanon: Saida, Hazmiye, Beirut, Nahr Brahim, Tripoli, Aabey, Aintoura, Bikfayia, Beit Meri, Raifoun, Feytroun, Jamhour, Falougha, Hasroun, Hadath, Ehden, Bcharre, Qoub Elias, Zahle, Chtaura, Rachaiya ( Mt Hermon region). - Hab: Among the rocks, red moist soil, sunny and shady places. - Uses: The sliced and crushed fresh bulb scales are locally applied for toothache and headache.

Ornithogalum lanceolatum Labill. - Syn : O. billardieri Mouterde - En: Labillardière's star-ofBethlehem. - Ar: Salsal Labiardyier. - Lf: Perennial herb, bulbous geophyte, 3-4 cm. - Ft: JanuaryFebruary. - Mt Hermon: 1290 m, 33²8’40”N, 3551'41'E. - Lebanon: Jabal El Barouk, Dahr Al Baidar, Jabal El Knisse, Jabal Sannine, Yammoune, Aaquora, Hasroun, Ainata, Zahle, Mt Hermon. - Hab: Humid soil among rocks. - Uses: Leaves and blossoms are boiled as depurative.

Ornithogalum libanoticum Boiss. - Syn: Honorius libanoticus (Boiss.) Holub - En: Lebanon star of Bethlehem. - Ar: Sasal Lubnani. - Lf: Perennial, bulbous geophyte, c. 15 cm. - Ft: April-May. -

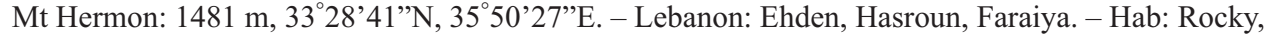
sunny slopes, red clay soil (terra rossa), medium mountain ranges. - Uses: Poisonous plant.

\section{Asteraceae (Compositae)}

Achillea falcata L. - En: Falcate milfoil. - Ar: Habbouk, Qaysum jabali. - Lf: Perennial herb, 1545 cm. -Ft: April-July. - Mt Hermon: 2074 m, 33²5’58’N, 35 50’20”'E. - Lebanon: Afqa, Mdeyrej, Sawfar, Bcharre, Ariz, Hasroun, Ehden, Dimane, Jabal El Knisse, Ain Bourdai, Baalbek, Rachaiya. - Hab: Rocky slopes, high mountain ranges, sunny places. - Uses: Medicinal plant used in the treatment of diabetes.

Anthemis rascheyana Boiss. - Syn: A. antilibanotica Eig - En: Rachaiya Chamomile. - Ar: Okhowan Rachaiya. - Lf: Annual herb, 5-15 cm. - Ft: March-May. - Mt Hermon: $1140 \mathrm{~m}$, $33^{\circ} 30^{\prime} 07^{\prime \prime} N$, 35 49'1'E. - Lebanon: Baalbek, Rachaiya (MtHermon region). Endemic to Lebanon and Syria. - Hab: Cultivated and abandoned lands, roadsides, pathways, olive groves, orchards, sunny places. - Uses: Decoction of flower heads is used as antiseptic and antidiabetic.

Carduus argentatus L. - En: Silvery Plumed Thistle. - Ar: Horshouf Foddi. - Lf: Annual herb, spiny, 30-50 cm. - Ft:April-May. - Mt Hermon: 1147 m, 3330’07’N, 35 49'41'E. - Lebanon: Tyr, Beirut, Hazmiyeh, Qalamoun, Nahr El Kalb, Aabey, Ghazir, Aintoura, Bhamdoun, Terbol, Arayia, Hadchit, Bcharre, Feytroun, Maghdouche, Akkar, Ksara. - Hab: Woodlands, open shrubby areas, meadows, roadsides, low mountain ranges, dry and damp places. - Uses: None documented.

Carlina libanotica Boiss. - Syn: C. involucrata subsp. libanotica (Boiss.) Meusel \& Kästner, C. corymbosa L. var. libanotica (Boiss.) Boiss., C. libanotica subsp. microcephala (Post) Meusel \& 
Dittrich - En: Involucrate Carline-thistle. - Ar: Zend Al Abd Al Lebnani. - Lf: Biennial herb, spiny, 10-80 cm. - Ft: June-September. - Mt Hermon 1325 m, 33²8'27’N, 35 50'39'E. - Lebanon: Ras Beirut, Ain Zhalta, Jabal Sannine, Jabal El Knisse, Ainata, Ammiq. - Hab: Dry stony mountainous regions, roadsides, pastures. - Uses: Decoction of roots is used as diuretic and in the treatment of skin rashes. The young stems are cleaned of spines and eaten boiled with legumes or fried in olive oil with lemon seasoning.

Carthamus tenuis (Boiss. \& C. I. Blanche) Bornm. subsp. tenuis - Syn: C. glaucus M. Bieb. var. tenuis (Boiss. \& C. I. Blanche) Boiss., Kentrophyllum tenue Boiss. \& C. I. Blanche - En: Thin Safflower. - Ar: Kortum Nahil. - Lf: Annual herb, spiny, 30-80 cm. - Ft: July-August. - Mt Hermon: 1322 m, 3327'52'N, 35 51'25”E. - Lebanon: Saida, Beirut, Tripoli, between Bikfayia and Antilias, Sin El Fil, Broumana, Bhamdoun, Souq El Gharb, Dimane, Qornayel, Bcharre. - Hab: Dry stony mountainous regions, roadsides, pastures, olive groves. - Uses: Roots extract is used to cure piles while seeds are believed to prevent cancer.

Centaurea drabifolia Sm. - Syn: Phaeopappus drabifolius (Sm.) Boiss. - En: Nailwort-leaved knapweed. - Ar: Qanturyun Libnan. - Lf: Perennial plant, $20 \mathrm{~cm}$. - Ft: June-August. - Mt Hermon: 2100 m, 3325'55'N, 35 50'15”E. - Lebanon: Jabal El Knisse, Hasroun, Dimane, Yammoune, Jabal Sannine, Qalaat Arrouba, Ariz, Makmel, Mt Hermon. - Hab: Stony slopes, amoung rocks, high mountain ranges, sunny and dry places. - Uses: Medicinal plant, decoction of aerial parts is used in the treatment of diabetes and wound healing.

Centaurea hyalolepis Boiss. - Syn: C. pallescens Delile var. hyalolepis (Boiss.) Boiss., C. pallescens subsp. hyalolepis (Boiss.) Holmboe - En: Transparent-bracted Knapweed. - Ar: Kantaryoun Shaffaf. - Lf: Annual herb, spiny, 60-100 cm. - Ft: April-July. - Mt Hermon: 1250 m, $33^{\circ} 28^{\prime} 54^{\prime}$ N, $35^{\circ} 51$ '35”E. - Lebanon: Saida, Beirut, Nahr Ibrahim, Mkalles, Zahle, Baalbek. - Hab: Dry and grassy grounds, roadsides, cultivated and abandoned lands. - Uses: Decoction of flowering parts is used as febrifuge.

Centaurea iberica subsp. hermonis (Boiss.) Bornm. - Syn: C. hermonis Boiss. - En: Iberian Knapweed, Star Thistle. - Ar: Chaoukat Al Dardar. - Lf: Annual herb, spiny, 20-80 cm. - Ft: MayJuly. - Mt Hermon: 1346 m, 33 28'54'N, 3551'47’'E. - Lebanon: Rachaiya, Hammara. - Hab: Dry and grassy habitats, roadsides, cultivated and abandoned lands. - Uses: The bitter astringent decoction of whole plant is used antidiabetic while the decoction of roots and fruits is used as diuretic for renal stones.

Cichorium intybus L. - Syn: C. intybus subsp. glabratum (C. Presl) Arcang. - En: Common Chicory. - Ar: Hendbe Barryye. - Lf: Perennial herb, 30-100 cm. - Ft: May-July. - Mt Hermon: 1300 m, 33 $26^{\circ} 10^{\prime}$ N, 35 50'21'E. - Lebanon: Dimane, Taanayel, Ksara. - Hab: Grassy grounds, fields, cultivated and abandoned lands, roadsides. - Uses: Decoction of leaves and roots is used as depurative, cholagogue, and for digestive troubles.

Cirsium leucocephalum subsp. hermonis (Boiss.) Greuter - Syn: C. lappaceum subsp. hermonis (Boiss.) Petr., C. hermonis Boiss. - En: Bur Thistle. - Ar: Kaswan Haramoun. - Lf: Perennial herb, spiny, 30-60 cm. - Ft: June-August. - Mt Hermon: 2417 m, 33²5'28'N, 35 50'57’'E. - Lebanon: Ariz, Jabal Sannine, Yammoune, Dimane, Ehden, Bcharre, Ainata, Marjhine, Rachaiya. Endemic to Lebanon and Syria. - Hab: Barren pasturelands, roadsides, mountainous regions. - Uses: edible (stalks are peeled and eaten raw) and melliferous plant. 
Cota palaestina Kotschy - Syn: Anthemis palaestina (Kotschy) Boiss. - En: Black-scaled Chamomile. - Ar: Okhouwan Aswad El Harashef. - Lf: Annual plant, 20-40 cm. - Ft: MarchAugust. - Mt Hermon: 1252 m, 33 30'01'N, 35 51'03'E. - Lebanon: Maghdouche, Aintoura, Ksara, Marjaayoun, Rachayia, Mt Hermon. - Hab: Meadows, pastures,damp places at low mountain ranges. - Uses: Medicinal plant, flower is used as antidiabetic, bacteriostatic and antibiotic.

Cousinia libanotica DC. - Syn: C. hermonis Boiss. - En: Hermon Cousinia. - Ar: Cousinia Haramon. - Lf: Spiny perennial herb, 20-80 cm. - Ft: June-August. - Mt Hermon: $1471 \mathrm{~m}$, $33^{\circ} 27^{\prime} 19$ 'N, $35^{\circ} 51$ '43'E. - Lebanon: Ainata, Deir El Ahmar, Baalbek, Ain Bourday, Ras Baalbek. Hab: Dry and rocky slopes, high mountain ranges, sunny places. - Uses: Extracts of aerial has properties against bacteria and fungi and is used in the treatment of anemia and bile. Dried whole plant is used as fuel.

Crepis libanotica J. Thiébaut - Syn: C. reuteriana subsp. eigiana Babc., C. reuteriana Boiss. var. alpina Boiss. - En: Reuter's Hawkweed. - Ar: Sraghet Lebnan. - Lf: Perennial herb, 30100 cm. - Ft: March-August. - Mt Hermon: 1250 m, 33²8'54”N, 35'51'35'E. - Lebanon: Saida, Beirut, Chekka, Al Qalamoun, Tripoli, Broumana, Chouit, Bikfayia, Tourza, Bzemmar, Beit Meri, Harissa, Raifoun, Jamhour, Aabey, Baabda, Mdeyrej, Jisr Al Hajar, Ariz, Dimane, Hasroun, Ain Halakim Rachaiya. - Hab: Grassy grounds, meadows, roadsides, low mountain ranges. - Uses: The infusion of flowers is recommended for the treatment of eye diseases and raw flowers are used in livestock feed.

Crepis robertioides Boiss. - En: Robertia Hawkweed. - Ar: Sraghet Robertiyya. - Lf: Perennial dwarf herb, 5-10 cm. - Ft: June-August. - Mt Hermon: 2306 m, 33²5’37'N, 35 50’32'E. Lebanon: Hasroun, Dimane, Yammoune, Dahr El Kadib, Makmel, Sannine, Qurnat as Sawda, Jabal El knisse, Rachaiya. Endemic to Lebanon and Syria. - Hab: Among rocks, old walls cracks, calcareous cliffs, roadsides among rubble, and barren, dry and sunny places. - Uses: The infusion of flowers is recommended for the treatment of eye diseases and raw flowers are used in livestock feed.

Crupina crupinastrum (Moris) Vis. - Syn: Centaurea crupinastrum Moris - En: False Sawwort. - Ar: Zahafa Hamraa. - Lf: Annual herb, 20-70 cm. - Ft: April-June. - Mt Hermon: 1250

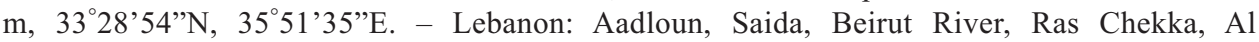
Qalamoun, Nahr Abou Ali, Baabda, Broumana, Bikfayia, Aabey, Terbol, Achkout, Jamhour, Hasroun, Bechnata, Akkar, Yammoune, Zahle. - Hab: Dry and stony hillslopes, abandoned lands, pastures, low mountain ranges, sunny places. - Uses: Decoction of aerial parts is potentially antiseptic and highly antimicrobial.

Echinops spinosissimus subsp. macrolepis (Boiss.) Greuter - Syn: E. viscosus subsp. macrolepis (Boiss.) Feinbrun, E. viscosus DC. var. macrolepis Boiss., E. syriacus Boiss. - En: Viscous Globe Thistle. - Ar: Chaouk Al Jamal. - Lf: Perennial herb, spiny, 80-100 cm. - Ft: May-August. - Mt Hermon: 1292 m, 3328'55'N, 3551'73’E. - Lebanon: Saida, Beirut, Aabey, Zahle, Jabal Sannine, Saadnayel, Taanayel, Beit Meri, Broumana, Souq El Gharb. - Hab: Dry and rocky slopes, scrub, roadsides, fields, abandoned lands, sunny places. - Uses: Decoction of root mixed with olive oil is used as hemostatic. Decoction of stems, leaves and roots is used as diuretic and for liver diseases.

Gundelia tournefortii L. - En: Tournefort's Gundelia, Tumbleweed, Tumble Thistle. - Ar: Akkub, Akoub. - Lf: Perennial, spiny herb, 20-50 cm. - Ft: April-May. - Mt Hermon: 1250 m, 3326'10 N, 35 50'21'E;

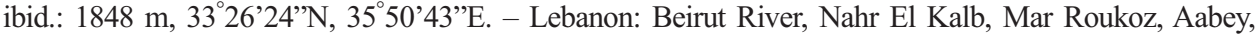
Tripoli, Bhamdoun, Ariz, Ehden, Akkar, Hasroun, Dahr Al Baidar, Ouadi El Harir. - Hab: Dry and rocky 
slopes, among mountain rocks, abandoned lands, sunny places. - Uses: Young stems and heads are cooked as a stew or egg-vegetable dish. Decoction of seeds, roots and stems is taken orally for colds, catarrh, diabetes, kidney pains. Latex is applied externally to treat vitiligo, edema and toothache.

Klasea pusilla (Labill.) Greuter \& Wagenitz - Syn: Serratula pusilla (Labill.) Dittrich, Cynara pusilla Labill., Rhaponticum pusillum (Labill.) Boiss., Rh. pygmaeum DC. - En: Dwarf Saw-wort. Ar: Warkha Kazama. - Lf: Perennial herb, 50-80 cm. - Ft: April-June. - Mt Hermon: $1282 \mathrm{~m}$,

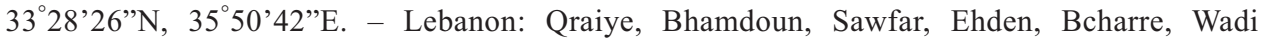
Qannoubine, Hasroun, Ain Zhalta, Saghbine, Sannine, Barrouk, Wadi El Harrir, Yammoune, Hazerta, Baalbek, Rachaiya. - Hab: Dry and rocky slopes, roadsides among rubble. - Uses: Extract of flowers isused as a repellent and an insecticide.

Matricaria recutita L. - Syn: Matricaria chamomilla L., nom. illeg. - En: Wild Chamomile. - Ar: Babunaj. - Lf: Annual herb, 10-40 cm. - Ft: March-April. - Mt Hermon: 1197 m, 33 29'47'N, 35 50'35'E. - Lebanon: Beirut, Tripoli, Jamhour, Hasbani. - Hab: fields, abandoned lands, low mountain ranges, sunny places. - Uses: Medicinal plant, decoction of aerial parts is orally used in the treatment of intestinal gasdiarrhea and other digestive system disorders.

Notobasis syriaca (L.) Cass. - Syn: Carduus syriacus L., Cirsium syriacum (L.) Gaertn. - En: Syrian Thistle. - Ar: Kharfeesh Kabeer. - Lf: Annual, spinyherb, 80-150 cm. - Ft: April-June. - Mt Hermon: 1185 m, 3329'17'N, 31 51'17'E. - Lebanon: Saida, Damour, Beirut, Tripoli, Aabey, Jamhour, Mazraat Beit Ech Chaar, Antoura, Zahle, Jabal El Barouk, Marjayoun, Rachaiya. - Hab: Cultivated and abandoned lands, stony hillsides, roadsides, low mountain ranges. - Uses: None documented.

Onopordum heteracanthum C. A. Mey. - Syn: O. anisacanthum Boiss., O. heteracanthum var. anisacanthum (Boiss.) Boiss., O. albolanatum Arènes, O. lanceolatum Eig, O. nudipes Arènes - En: Cotton thistle. - Ar: Aksoun Chaek. - Lf: Biennial herb, spiny, $100 \mathrm{~cm}$. - Ft: April-July. - Mt Hermon: 1292 m, 3328'54'N, 3551'35'E. - Lebanon: Baalbek, Zahle, Saadnayel, Nahr El Aassi. - Hab: Rocky and stony grounds, abandoned lands, field boundaries, roadsides. - Uses: Young stems are striped of spines and mostly cooked or boiled with others vegetables and fruits.

Picnomon acarna (L.) Cass. - Syn: Carduus acarna L., Cirsium acarna (L.) Moench - En: Yellow Cnicus. - Ar: Kaswan Al Jamal. - Lf: Annual herb, spiny, 50-100 cm. - Ft: July-August. - Mt Hermon: $1191 \mathrm{~m}, 33^{\circ} 28^{\prime} 55 \mathrm{~N}, 35^{\circ} 51^{\prime} 73$ E. - Lebanon: Beirut, Broumana, Qraiaa, Aabey, Bhamdoun, Dimane, Khan Sannine, Mreijat, Taanayel, Ksara. - Hab: Dry and rocky slopes, cultivated and abandoned lands, roadsides. - Uses: Decoction of aerial parts is used as treatment of gastric pain and rheumatism. When young, this thorny plant can be eaten by animals. The large hard branches are used as fuel for ovens and hearths.

Podospermum canum C. A. Mey. - Syn: Scorzonera cana (C. A. Mey.) Griseb., S. euphratica Gomb. \& Arènes; the genus Podospermum, as different from Scorzonera, is well supported by molecular phylogenetic analyses (Mavrodiev \& al. 2004). - En: White Viper's Grass. - Ar: Dabeh Jabali. - Lf: Perennial herb, 5-50 cm. - Ft: April-August. - Mt Hermon: 1250 m, 33 28'54'N, 3551'35'E. - Lebanon: Jabal El Knisse, Jabal Sannine, Baskinta, Ain Zhalta, Hasroun, Tannourine, Yammoune, Chtaura, Taanayel, Rayak, Sir Al Danniyeh, Baalbek, Jabal Makmel, Qurnat as Sawda. - Hab: Rocky and grassy grounds, old walls cracks, cliffs and roadsides among rubble. - Uses: Decoction of aerial parts is used orally to treat headaches, raw young shoots and leaves are edible. 
Podospermum phaeopappum Boiss. - Syn: Scorzonera phaeopappa (Boiss.) Boiss. - En: Greypappused viper's grass. - Ar: El Meshe. - Lf: Perennial herb, 20-40 cm. - Ft: April-May. - Mt Hermon: 1147 m, 33 30'07'N, 35 49'41'E. - Lebanon: Baalbek, Machghara, Aita El Foukhar, Yanta, Hasbaya. - Hab: Rocky slopes, roadsides, pastures at low mountain ranges. - Uses: Raw aerial parts are edible and used to relief headaches.

Rhagadiolus stellatus (L.) Gaertn. - Syn: Rh. stellatus var. hebelaenus DC., Rh. stellatus var. edulis (Gaertn.) DC., Rh. edulis Gaertn. - En: Edible Hawkbit. - Ar: Raghad Youlous. - Lf: Annual herb, 20-60 cm. - Ft: March-June. - Mt Hermon: 1252 m, 33³0’01'N, 35 51'03”'E. - Lebanon: Saida, Beirut, Ras Chekka, Faraiya, Mrouj, Ras El Harf, Ehden, Knisse. - Hab: Cultivated and abandoned lands, hillslopes, stony ground, low mountain ranges, dry and sunny places. - Uses: None documented.

Scolymus hispanicus L. - En: Golden Thistle, Spanish Oyster Plant. - Ar: Shawkat Al Far. - Lf: Herbaceous biennial or perennial plant, spiny, 50-100 cm. - Ft: May-September. - Mt Hermon: 1250 m, 3328'54'N, 35 51'35'E. - Lebanon: Tyre, Hadath, Beirut, Dbaiye, Tripoli, Kadisha, Ghazir. Hab: Cultivated and abandoned lands, roadsides, sunny places. - Uses: The midribs are boiled and eaten as artichokes.

Scorzonera libanotica Boiss. - En: Lebanon Viper's Grass. - Ar: Dabh Lebnani. - Lf: Perennial herb, 15-80 cm. - Ft: June-July. - Mt Hermon: 1197 m, 3329'47’N, 3550’35'E. - Lebanon: Bcharre, Ariz, Ehden Makmel, Falougha, Marjhine. Endemic to Lebanon and Syria. - Hab: Rocky and grassy grounds, meadows, old wall cracks, cliffs, rubble by roadsides. - Uses: Decoction of aerial parts is used orally to treat headaches, raw young shoots and leaves are edible.

Tanacetum densum (Labill.) Sch. Bip. subsp. densum - Syn: Pyrethrum densum Labill., Chrysanthemum densum (Labill.) Steud. - En: Dense Tansy. - Ar: Tanastom Kaseef. - Lf: Perennial herb, $50 \mathrm{~cm}$. - Ft: June-August. - Mt Hermon: $2301 \mathrm{~m}, 33^{\circ} 35^{\prime} 38^{\prime}$ N, 35 50'30'E. - Lebanon: confined to Mt Hermon area. - Hab: Stony red clay soil (terra rossa), high mountain ranges among rocks. - Uses: Infusion of aerial parts is locally used for skin diseases, hygiene and vulnerary forwounds.

Taraxacum assemanii Boiss. - Syn: T. primigenium Hand.-Mazz. - En: Assemani’s Dandelion. - Ar: Tarkhachkoon Al Samaiina. - Lf: Perennial herb. - Ft: July-August. - Mt Hermon: 1475 m, $33^{\circ} 28^{\prime} 46^{\prime}$ N, 35 50'21'E. - Lebanon: Amioun, Dimane, Hasroun, Qurnat as Sawda, Jabal Sannine, Jabal El Knisse, Jabal Makmel. - Hab: Calcareous cliffs, among rocks, grassy grounds, rubble by roadsides. - Uses: Raw leaves and roots are edible as a salad with olives, onions and bread.

Taraxacum phaleratum G. E. Haglund - En: Shiny Dandelion. - Ar: Tarkhachoun Lamee. - Lf: Perennial herb. - Ft: April-May. - Mt Hermon: 1140 m, 33³0'07'N, 3549'41'E. - Lebanon: Hermel, Halba, Ain Aata (Mt Hermon region). - Hab: Cultivated and abandoned lands, among rocks, grassy grounds, rubble by roadsides. - Uses: Leaves and roots are edible raw or cooked, and used raw in salads dressed with olive oil, lemon juice or vinegar.

Tragopogon buphthalmoides var. latifolius Boiss. - Syn: T. brevifolius K. Koch, T. plantagineus Boiss. \& A. Huet - En: Oxeye Goat's Beard. - Ar: Ain El Thaour. - Lf: Perennial herb, 20-50 cm. Ft: April-July. - Mt Hermon: 1250 m, 3328'54'N, 35'51'35'E. - Lebanon: Barouk, Bhamdoun, Raifoun, Zahle, Sannine, Laqlouq, Tannourine, Hasroun, Dimane, Hadath, Makmel, Ariz, Taanayal, Ksara, Rachaiya. - Hab: Dry and rocky slopes, grassy grounds, rubble by roadsides. - Uses: Infusion 
of aerial parts is used orally to treat gastro-intestinal disorders as vermifuge and for heart diseases. Young leaves, root and stems are edible raw or boiled.

Tragopogon porrifolius subsp. longirostris (Sch. Bip.) Greuter - Syn: T. longirostris Sch. Bip. En: Long Beaked Goat's Beard. - Ar: Lehyat El Tays. - Lf: Biennial herb, 30-50 cm. - Ft: AprilJuly. - Mt Hermon: 1292 m, 3328'55’N, 3551'37’E. - Lebanon: Saida, Beirut, Nahr El Kalb, Tripoli, Broumana, Douma, Bikfayia, Bcharre, Ksara, Barouk, Chtaura. - Hab: Dry and rocky slopes, grassy grounds, rubble by roadsides. - Uses: Infusion of aerial parts is used orally to treat gastrointestinal disorders, heart disease and as vermifuge. Young leaves, root and stems are edible raw or boiled.

\section{Berberidaceae}

Bongardia chrysogonum (L.) Spach - Syn: B. rauwolfii C. A. Mey., Leontice chrysogonum L. - En: Golden Rod. - Ar: Orf El Deek. - Lf: Perennial herb, 20-80cm. - Ft: February-April. - Mt Hermon: 1140 m, 3330'07'N, 3549'41'E. - Lebanon: Tyre, Fayadiye, Bhamdoun, Kartaba, Daher el Baydar, Qana near Tyre, Jabal El Knisse, Bar Elias, Taanayel, Mreijat to Chtaura and Taalabaya, Rachaiya. - Hab: Meadows, red gravel soil, vineyards, rock-crevices, old walls, dry and sunny places. - Uses: Rhizomes and leaves are edible (taste of lemon). The extract of plant and roots is used as anti-inflammatory, anticancer, and in the treatment of haematopoietic neoplasms. Extract of tubers is used in epilepsy. Chilled decoction of tubers $\left(2-3^{\circ} \mathrm{C}\right)$ is believed to treat urinary tract, prostate disorders and haemorrhoids.

\section{Boraginaceae}

Alkanna orientalis (L.) Boiss. - Syn: A. hellenica (Boiss.) Rech. f., A. leucantha Bornm., Anchusa orientalis L., Lithospermum orientale (L.) L. - En: Oriental Alkanet, Yellow Alkanet. - Ar: Shenjar Sharki. - Lf: Perennial herb, 20-50 cm. - Ft: March-June. - Mt Hermon: 2338 m, 33 25'36”N, 35 50'37'E. - Lebanon: Ariz, Bcharre, Hadath, Baalbek, Rachaiya. - Hab: Abandoned lands, limestone gravel soil, high mountain ranges, dry and sunny places. - Uses: Stem bark is used as astringent and taken orally in the treatment of ulcers and other intestinal diseases.

Anchusa hybrida Ten. - Syn: Anchusa undulata subsp. hybrida (Ten.) Bég. - En: Hybrid Bugloss. - Ar: Lisan Al Thaour. - Lf: Perennial herb, 20-50 cm. - Ft: Feb-June. - Mt Hermon: 1292 m, $33^{\circ} 28^{\prime} 54^{\prime}$ N, 35 51'35”E. - Lebanon: Saida, Tripoli, Beirut, Bir Hassan, Ouzai, Beirut River, Machgara, Terbol, Sannine, Dahr Al Baidar, Ain Zhalta, Hasroun, Qannoubine, Ehmej, Hadath, Akkar, Ebel Es Saqi. - Hab: Cultivated lands, fallow grounds, open shrubby areas, dry hillslopes, low mountain ranges. - Uses: Edible, medicinal, odoriferous and melliferous plant. Used as diuretic and in the relief of cough, muscular pains, rheumatism and stomachache.

Brunnera orientalis (Schenk) I. M. Johnst. - Syn: Myosotis orientalis Schenk, Anchusa neglecta A. DC. - En: Oriental Brunnera. - Ar: Brunera Sharkiyya. - Lf: Perennial herb, 30-60 cm. - Ft: March-May. - Mt Hermon: 1843 m, 3326'24'N, 3550'48”E. - Lebanon: Maaser, Jabal Kneise, Zahle, Faraya, Ehden, Qannoubine, Hasroun, Ariz, Jabal Sannine, Sir Al Danniyeh, Afqa, Ouadi El Harir, Baalbek, Dimane, Bkaa Kafra, Bcharre, Akkar, Ainata, Rachaiya. - Hab: Rocky slopes, high mountain ranges, dry and shady places. - Use: Ornamental plant.

Onosma sericea Willd. - En: Silky Golden-drop. - Ar: Onosma Naaima. - Lf: Perennial herb, 40-

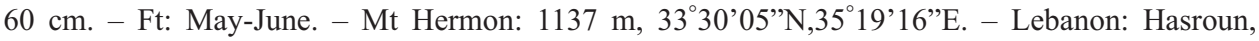


Qannoubine, Dimane, Ehden, Jabal Niha, Qoub Elias, Ksara, Bcharre, Bqaa Kafra, Deir El Aachayer, Zahle, Kfar Zabad, Baalbek, Rachaiya. - Hab: Grassy grounds, shady places, mountain rocks, low mountain ranges. - Uses: Medicinal plant used as a laxative and to treat bronchitis, wounds, snake bites, burns and urinary calculi.

\section{Brassicaceae (Cruciferae)}

Alyssum condensatum subsp. flexibile (Nyár.) T. R. Dudley - Syn: A. flexibile Nyár., A. venustum Nyár. - En: Condensed Mad-wort. - Ar: El Wasan El Mondamej. - Lf: Annual herb, 5- 15 cm. - Ft: May-August. - Mt Hermon: 1575 m, 3327'21'N, 3552'42”E. - Lebanon: Jabal El Knisse, Qozhaiya, Hasroun, Tannourine, Barouk, Jabal Sannine, Dahr El Kadib, Makmel, Markaa Ej Jamal, Qurnat as Sawda. - Hab: Quercus woodlands, dry and rocky slopes, roadsides. - Uses: None documented.

Arabis alpina L. - Syn: A. alpina subsp. caucasica (Willd.) Briq., A. caucasica Willd. - En: Caucasian Rockcress. - Ar: Arabi Kofkas. - Lf: Perennial plant, 15-35 cm. - Ft: April-July. - Mt Hermon: 1808 m, 3326'41'N, 35 50'32”E. - Lebanon: Mdeyrej, Jabal El Knisse, Laqlouq, Maasser Al Chouf, Hasroun, Hadchit, Jabal Sannine, Tannourine, Aaqoura, Faraiya, Ariz, Yammoune, Ainata, Deir El Aachayer, Rachaiya. - Hab: Calcareous rocks, high mountain ranges, dry and sunny places. - Uses: Edible plant (flowers and leaves).

Fibigia clypeata (L.) Medik. - Syn: Fibigia rostrata (Schenk) Boiss., Fibigia obovata Boiss., Farsetia clypeata (L.) W. T. Aiton - En: Shield Fibigia. - Ar: Hasheeshat El Kouna. - Lf: Perennial herb, 30-75 cm. - Ft: March-May. - Mt Hermon: 1182 m, 33 29'59”N, 3549'52"E. Lebanon: Qraiaa, Hasroun, Hadchit, Sannine, Hazerta, Baalbek, Rachaiya. - Hab: Scrub, rocky slopes, gravelly non-calcareous soils, low mountain ranges. - Uses: Medicinal plant administered as a diuretic.

Sisymbrium officinale (L.) Scop. - Syn: Chamaeplium officinale (L.) Wallr. - En: Hedged mustard. - Ar: Sumara Tobeyia. - Lf: Annual plant, 5-90 cm. - Ft: Spring. - Mt Hermon: 1197 m, 3329'47’N, 35'50'35'E. - Lebanon: Beirut, Batroun, Tripoli, Aabey, Harissa, Qannoubine, Akkar, Chtaura, Zahle, Hasbani. - Hab: fields, meadows, roadsides. - Uses: Medicinal plant used to soothe sore throat, laxative, diuretic and antiseptic.

\section{Campanulaceae}

Campanula rapunculus L. - En: Rampion. - Ar: Jrays Sanabli. - Lf: Perennial herb, 40-80 cm. - Ft: April-May. - Mt Hermon: 1410 m, 33²9'14”N, 3551'10”E. - Lebanon: Saida, Naher E1 Damour, Choueifat, Beirut, Beirut River, Nahr El Kalb, Ras Chekka, Tripoli, Raifoun, Harissa, Beit Meri, Mairouba, Hammana, Hadath, Hadchit, Zahle, Qalaat El Chekif, Rachaiya. - Hab: Dry and sunny meadows, medium mountain ranges. - Uses: Raw or cooked fresh leaves and roots are edible. Plant distilled in water is useful as digestive stimulant, in diabetes, facial treatment and skin whitening.

Legousia falcata (Ten.) Janch. - Syn: Prismatocarpus falcatus Ten., Specularia falcata (Ten.) A. DC. - En: Legousie falciforme. - Ar: Lighuzyah Minjaliyyah. - Lf: Annual plant, about $30 \mathrm{~cm}$. - Ft: AprilMay. - Mt Hermon: 1224 m, 3329'08’N, 3551'29'E. - Lebanon: Ras Damour, Baabda, Beirut, Antilias, Nahr El Kalb, Khalde, Beirut River, Ras Chekka, Tripoli, Ghazir, Aabey, Faitroun, Qrayye, 
Bikfayia, Qannoubine, Ehmej, Dahr Al Baidar, Afqa, Zahle, Rachaiya. - Hab: Stony pastures, hill slopes, roadsides, low mountain ranges, sunny places. - Uses: Ornamental plant.

Legousia pentagonia (L.) Druce - Syn: Campanula pentagonia L., Specularia pentagonia (L.) A. DC. - En: Pentagonal legousia. - Ar: Lighouziya Khomasia. - Lf: Perennial herb, 30 cm. - Ft: AprilJune. - Mt Hermon: 2338 m, 3325’36”N, 3550'37’E. - Lebanon: Qadisha, Aabey, Maasser El Chouf, Tourzaiya, Ehden, Bcharre, Akoura, Sannine, Ain Zhalta, Niha, Marjayoun, Qoub Elias, Ammiq, Haouch Al Oumara, Bazoun, Hasroun, Yammoune, Chlifa, Zahle, Meksi, Baalbek, Rachaiya. - Hab: Cultivated and abandoned lands, occasionally on open limestone hillsides at high altitudes.- Uses: None documented.

\section{Capparaceae}

Capparis sicula Veill. subsp. sicula - Syn: C. spinosa L. var. canescens Coss. - En: Common Caper, Caper Berries. - Ar: Kobar. - Lf: Shrub, 50-150 cm. - Ft: May-August. - Mt Hermon: 1197 m, $33^{\circ} 29^{\prime} 47^{\prime \prime N}, 35^{\circ} 50^{\prime 3}$ '”E. - Lebanon: Saida, Beirut, Nahr El Kalb, Tripoli, Zahle, Hasbay; highly abundant at lower altitudes starting from $800 \mathrm{~m}$ but dispersed individuals observed at higher altitudes with 1197 m reported here being the uppermost occurrence in Lebanon so far. - Hab: Rocky places, cliffs and walls. - Uses: The flower buds are pickled in oil or vinegar and used as flavouring in sauces, salads. Decoction of barks and roots is taken orally as diuretic, astringent and to treat gout and rheumatism. Infusion of fruits and leaves is carminative and aphrodisiac. Decoction of flower buds is also taken for rheumatism while the infusion of fruits is used for sciatica and rheumatism.

\section{Caprifoliaceae}

Lonicera nummulariifolia Jaub. \& Spach - En: Nummular-leaved honeysuckle. - Ar: Lunisirah naqdiyyah al waraq. - Lf: Shrub, 60-120 cm. - Ft: June-July. - Mt Hermon: 1508 m, 33²7'10'N, 35 50'28"E. - Lebanon: Ariz El Maasser, Jabal El Barouk, Ariz Ain Zhalta, Tannourine, Rahoue, Ariz, Qadisha, Ehden, Ainata, Yammoune, Rachaiya, Hasbaya, Kfar Zabad. - Hab: rocky slopes, among rubble, sunny places. - Uses: Ornamental plant.

\section{Caryophyllaceae}

Dianthus strictus subsp. multipunctatus (Ser.) Greuter \& Burdet - Syn: D. multipunctatus Ser., D. polycladus Boiss., D. strictus subsp. polycladus (Boiss.) Kollmann - En: Wild Pink. - Ar: Koronfol Moutaadid Al Boukaa. - Lf: Perennial herb, 15-80 cm. - Ft: May-July. - Mt Hermon: 1250 m, 3328'54”N, 3551'35'E. - Lebanon: Saida, Al Awali River, Beirut, Beirut River, Antilias, Mkalles, Nahr El Kalb, Jounieh, Tripoli, Broumana, Bikfayia, Qraiye, Hammana, Aabey, Mairouba, Ain Zhalta, Sawfar, Knisse, Ehmej, Ariz, Ehden, Dimane, Hasroun, Taanayel, Ksara, Kefraiya, Baalbek, Ouadi El Harir. - Hab: Grazing fields, dry and rocky slopes, sunny places. - Uses: Plant is used traditionally to treat teeth inflammations.

Minuartia juniperina (L.) Maire \& Petitm. - Syn: Alsine juniperina (L.) Wahlenb. - En: Juniper Sanwort. - Ar: Minurieta Arareyya. - Lf: Annual plant, 3-12 cm. - Ft: May-July. - Mt Hermon: 2102

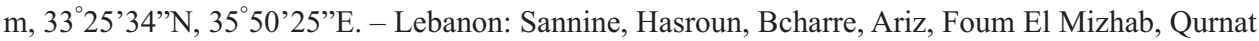
as Sawda, Sir Al Danniyeh, Rachaiya. - Hab: Calcareous rocks, cervices, high mountain ranges. Uses: Ornamental plant. 
Silene aegyptiaca (L.) L. - Syn : S. atocion Jacq., S. atocioides Boiss., Cucubalus aegyptiacus L. Enatchfly. - Ar : Shentan El Noreyyi. - Lf: Annual herb, 15-20 cm. - Ft: January-April. - Mt Hermon: 1140 m, 33 30'07'N, 35 49'41'E. - Lebanon: Faiyadiyeh, Aley, Bcheale, Hasroun, Ebel Es Saqi, Rachaiya. - Hab: Fields, roadsides, waste places. - Uses: Fumigation with seeds is used to cure sore throat.

Silene vulgaris (Moench) Garcke - Syn: S. cucubalus Wibel, S. inflata Sm., S. latifolia Poir., Silene venosa Asch. En: Common Campion. - Ar: Silinah Mabdhulah. - Lf: Perennial herb, 30-60 cm. Ft: February-June. - Mt Hermon: 1220 m, 33²8’31'N, 3551'10”'E. - Lebanon: Saida, Beirut, Nahr El Kalb, Tripoli, Jounieh, Bikfaya, Aabey, Beit Meri, Asfouriye, Dahr Al Baidar, Berkacha, Ariz, Hasroun, Dimane, Sarada, Jisr El Khardali. - Hab: fields, pastures, low mountain ranges, sunny places. - Uses: Young shoots and leaves are edible raw or cooked.

\section{Chenopodiaceae (Amaranthaceae s. 1.)}

Blitum virgatum L. - Syn: Chenopodium virgatum (L.) Ambrosi non Thunb., Ch. foliosum Asch., Morocarpus foliosus Moench; for generic concept see Fuentes-Bazan \& al. (2012). - En: Leafy Goosefoot. - Ar: Rejl El Waz. - Lf: Annual herb, 50 cm. - Ft: May-July. - Mt Hermon: 2075 m, $33^{\circ} 25^{\prime} 58^{\prime}$ N, $35^{\circ} 50^{\prime} 02^{\prime \prime E}$. - Lebanon: Niha, Jabal El Barouk, Jabal El Knisse, Faraiya, Aayoun Al Simane, Bcharre, Ariz, Jabal Sannine, Jroud Hasroun, Qurnat as Sawda. - Hab: High mountain ranges, rocky slopes, sunny places. - Uses: Fruits are used fresh for dyspnoea.

Chenopodium vulvaria L. - En: Stinking Goosefoot. - Ar: Sarmak Krayye. - Lf: Annual plant, 1560 cm. - Ft: July-Sept. - Mt Hermon: 1213 m, 3329’10”N, 35'51'26”E. - Lebanon: Saadiyat, Beirut, Tripoli, Qraiaa, Broumana, Aabey, Douma, Bcharre, Dimane, Zahle, Marjayoun, Baalbek. Hab: Walls, roadsides, on plant debris and animal waste, grazed land, dry and bare soil. - Uses: Medicinal plant, aerial parts are used as analgesic, vermicide and in the treatment of abdominal pains, flatulence and diarrhea.

Noaea mucronata subsp. humilis (Boiss.) Danin \& Raus - Syn: Noaea spinosissima (L. f.) Moq. var. humilis Boiss., Noaea mucronata (L. f.) Moq. var. humilis (Boiss.) Dinsm. -En: Dwarf Mucronate Noaea. - Ar: Shawk El Hanash Sagheer. - Lf: Dwarfshrub, 20-30cm. - Ft: July-August. - Mt Hermon: 2417 m, 3325'57’N, 35 50’30”E. - Lebanon: Ariz, Bcharre, Jabal Sannine, Yammoune, Qurnat as Sawda, Ehden, Mt Hermon. - Hab: Rocky slopes, dry and sunny sites of high mountain ranges (for ecological and chorological details see Greuter \& Raus 2012: 289). - Uses: None documented.

\section{Clusiaceae (Guttiferae)}

Hypericum libanoticum N. Robson - Syn: H. helianthemoides auct. non (Spach) Boiss., H. hyssopifolium auct. non Chaix, Drosanthe helianthemoides auct. non Spach - En: Lebanon Saint John'swort. - Ar: Dazii Lebnani. - Lf: Perennial herb, up to $60 \mathrm{~cm}$. - Ft: May-August. - Mt Hermon: 1925 m, 3326'17'N, 35 50'37'E. - Lebanon: Chouf, Jabal El Barouk, $1800 \mathrm{~m}$ above Ain Zhalta, Ariz, Jabal Sannine, Yammoune, Zahle, Ouadi El Arayech, Ouadi El Harir. Endemic to Lebanon and Syria. - Hab: Dry and gravel soil, among mountain rocks, high mountain ranges, sunny places. - Uses: Infusion of flowering parts is used as diuretic, vermifuge, astringent and vulnerary.

Hypericum triquetrifolium Turra - Syn: Hypericum crispum L., nom. illeg. - En: Tumble St. John's Wort. - Ar: Dazi Mosallas El Warak. - Lf: Perennial herb or subshrub, 20-30 cm. - Ft: May-Sept. - 
Mt Hermon: 1364 m, 33²8’29'N, 35 51'06”E. - Lebanon: Saida, Beirut, Antilias, Madfoun, Tripoli, Kahale, Ghosta, Zahle, Mt Hermon. - Hab: Dry scrub, rocky cliffs, roadsides, mountain pastures, open shrubby areas, low mountain ranges, sunny places. - Uses: Medicinal plant, flower infusion is orally used as a sedative, depurative, astringent and to relieve headache. Plant is recognized as toxic for livestock.

\section{Convolvulaceae (incl. Cuscutaceae)}

Convolvulus libanoticus Boiss. - En: Lebanon bindweed. - Ar: Lablab lubnani. - Lf: Perennial herb, 5-30 cm. - Ft: July-August. - Mt Hermon: 1321 m, 33 27'49”N, 35 51'12”E. - Lebanon: Knisse, Jabal El Barouk, Jabal Sannine, Hasroun, Dimane, Yammoune, Ariz, Tannourine, Hermon summit. Endemic to Lebanon and Syria. - Hab: roadsides, meadows, sunny places. - Uses: None documented.

Cuscuta balansae Yunck. - Syn: C. globulosa Boiss. \& Reut. non Benth., C. palaestina subsp. balansae (Yunck.) Plitmann - En: Balansa's Dodder. - Ar: Kachout Balansa. - Lf : Parasitic herb. Ft: June-August. - Mt Hermon: 1364 m, 33²8'29’N, 35 51'06”E. - Lebanon: Hasroun, Bcharre, Mar Semaane, Jabal Sannine, Jabal El Barouk, Dimane, Yammoune, Bouarij, Hazerta, Jabal El Knisse, Baskinta, Afqa. - Hab: Parasitic on a great variety of herbaceous plants of fields and grassy grounds. - Uses: Decoction of plant is used as laxative and for jaundice.

\section{Crassulaceae}

Rosularia libanotica (Labill.) Muirhead - Syn: Cotyledon libanotica Labill., Umbilicus libanoticus (Labill.) DC. - En: Lebanon Navel-wort. - Ar: Wardeyyet Lebnan. - Lf: Perennial herb, 30-50 cm. - Ft: May-July. - Mt Hermon: 1432 m, 3328'46”N, 35'50'36”E. - Lebanon: Ariz, Ouadi El Arayech, Jezzine, Ghazir, Jabal El Barouk, Ain Zhalta, Jabal El Knisse, Ehden, Hasroun, Arbet Qozhaiya, Makmel, Yanta. - Hab: Chasmophyte of calcareous cliffs, crevices and hedges, usually in shade, at medium and high altitudes. - Uses: decoction of aerial parts is used as diuretic and applied locally in the treatment of suppurative skin inflammation and carbuncles.

Sedum amplexicaule subsp. tenuifolium (Sm.) Greuter - Syn: Sedum tenuifolium (Sm.) Strobl, Sempervivum tenuifolium Sm. - En: Slender-leaved stonecrop. - Ar: Hayyoun Aasfar. - Lf: Perennial herb, 7-20 cm. - Ft: April-June. - Mt Hermon: 1200 m, 33²9'16”N, 3551'08'E. - Lebanon: Bikfaya, Douma, Qraye, Machghara, Ain Hasroun, Hadath, Dimane, Ain Dahab, Ainata, Zahle, Hazerta, Zahle, Baalbek. - Hab: Rocky slopes, among debris and rocks, old walls. - Uses: None documented.

Umbilicus intermedius Boiss. - En: Common Penny-wort. - Ar: Asa El Raai. - Lf: Perennial herb, 20-60 cm. - Ft: March-June. - Mt Hermon: 1243 m, 3328'40”N, 35'51'41'E. - Lebanon: Dammour, Beirut, Tripoli, Ghazir, Bikfayia, Jabal Terbol, Broumana, Kahale, Ehden. - Hab: Rockcrevices, cliffs and old walls, mountains, sunny and shady places. Uses: Fresh crushed leaves are used in the treatment of suppurative skin inflammation and carbuncles.

\section{Cucurbitaceae}

Bryonia multiflora Boiss. \& Heldr. - Syn: B. macrophylla Boiss., B. lasiocarpa Mouterde - En: Many Flowered Bryony. - Ar: Inab Al Hayya. - Lf: Perennial herbaceous climber, tuberous 
geophyte, up to 4 m. - Ft: February-May. - Mt Hermon: 1290 m, 33²3'06’N, 35 51'18'E. Lebanon: Saida, Beirut, Nahr El Kalb, Tripoli, Bikfayia, Aabey, Broumana, El Barouk, Raifoun, Chtaura, Taalabaya, Qoub Elias, Aitanit, Qraiaa, Beit Meri, Maalaqa, Rachaiya. - Hab: Scrubs, hedges, woodland margins, generally on calcareous soil. - Uses: Decoction of fresh roots is purgative and externally used in rheumatism and itching.

Ecballium elaterium (L.) A. Rich. - Syn : Momordica elaterium L. - En: Squirting Cucumber. - Ar: Faaous Al Himar, Khiyar Al Himar. - Lf: Perennial herb, tuberous, 15-60 cm. - Ft: April-December. - Mt Hermon: 1197 m, 33²9'47’N, 3550’35”E. - Lebanon: Beirut, Tripoli, Broumana, Qraiye, Ksara. - Hab: Dry areas with rubble and stones, roadsides, abandoned lands, field margins. - Uses: The plant is toxic at very low doses. The fruit juice used as nose drops by nasal instillation for jaundice (once a day for one week). The root and fruit juice is locally used against rheumatism and edema.

\section{Cupressaceae}

Cupressus sempervirens L. - En: Graveyard Cypress, Pencil Pine. - Ar: Sharbeen, Sarow. - Lf: Evergreen tree, 12-22 m. - Ft: March-May. - Mt Hermon: 1200 m, 3329'19'N, 3551'04'E, naturalized. - Lebanon: Maghdouche, Deir Al Qamar, Aabey, Maamelteine, Ghazir, Jbail, Bcharre, Ehden. - Hab: Rocky slopes, gardens, several habitats, several mountain ranges. - Uses: Cones and wood are used as sedatives and cough expectorant. Essential oil is deodorant and antiseptic and in the treatment of acne and to relief footache and piles.

\section{Dennstaedtiaceae}

Pteridium aquilinum (L.) Kuhn - Syn: Pteris aquilina L. - En: Bracken, Eagle Fern, Northern Bracken Fern. - Ar: Khenshar El Okab. - Lf: Tall perennial herb, 30-300 cm. - Mt Hermon: 2057 m, 3326'10”N, 3550’21'”. - Lebanon: Dekwaneh, Arayia, Aabey, Bikfayia, Mrouj, Mairouba, Maghdouche, Faraiya, Qamouaa, Kobayat. - Hab: Mountain rocks, crevices, red clay soil, roadsides, forests, dry and warm places at medium and high altitudes. - Uses: The rhizomes of this fern are externally used as analgesic, but may cause dermatitis in sensitive individuals. The fronds and fiddlenecks can be toxic to cattle and horses.

\section{Dipsacaceae}

Cephalaria setosa Boiss. \& Hohen. - En: Bristly Cephalaria. - Ar: Zwan Shaouki. - Lf: Annual herb, 30-60 cm. - Ft: June-July. - Mt Hermon: 1620 m, 33²6’49'N, 35 50'33'E. - Lebanon: Falougha, Saadnayel, Ammiq, Wadi Fouara-Jezzine, Ouadi El Harir. - Hab: Meadows, rockcrevices, roadsides, cultivated and abandoned lands, dry and sunny places. - Uses: Juice of fresh stems is applied locally as antihaemorrhage and for wound healing.

Cephalaria stellipilis Boiss. - En: Starhaired Cephalaria. - Ar: Zwan Najme El Wabar. - Lf : Perennial herb, 20-80 cm. - Ft: July-October. - Mt Hermon: 1474 m, 33 25'45”N, 35 50'25”'E. Lebanon: Deir Al Qamar, Ain Zhalta, Jabal El Barouk, Yammoune, Ainata, Hammara. - Hab: Rocky slopes, gravel soil, dry and sunny places at medium altitudes. - Uses: None documented.

Lomelosia argentea (L.) Greuter \& Burdet - Syn: Scabiosa argentea L., S. ucranica L. - En: Silvery Scabious. - Ar: Jarbeyya Fodeyya. - Lf: Biennial or perennial herb, 50-80 cm. - Ft: May-

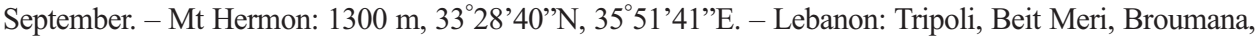


Bikfayia, Qraiye, Moukhtara, Laqlouq, Dimane, Hasroun, Ehmej, Bcharre, Jabal El Barouk, Qamouaa. Hab: Rocky slopes, mountain pastures, fields, dry and sunny places.- Uses: None documented.

Lomelosia divaricata (Jacq.) Greuter \& Burdet - Syn: Scabiosa sicula L. - En: Sicilian Scabious. - Ar: Jarbeyyet Sikleyia. - Lf: Annual plant, 10-30 cm. - Ft: March-April. - Mt Hermon: 1486 m, $33^{\circ} 27^{\prime} 29^{\prime \prime}$ N, 35 52'22'”E. - Lebanon: Mar Roukoz, Ksara. - Hab: Dry and rocky slopes, mountain pastures, fields, medium mountain ranges, dry and sunny places. - Uses: Medicinal plant, aerial part is used as a diuretic and in the treatment of scabies and wounds healing.

Lomelosia palaestina (L.) Raf. - Syn: Scabiosa palaestina Boiss. - En: Palestine Scabious. - Ar: Jarbeyya Falastinyie. - Lf: Annual herb, 20-100 cm. - Ft: March-April. - Mt Hermon: $1250 \mathrm{~m}$, $33^{\circ} 28^{\prime} 54^{\prime \prime}$ N, 35 51'35'E. - Lebanon: Tannourine, Bhamdoun, Deir El Harf, Ouadi El Harir, Deir El Ahmar, Qoubaiyat. - Hab: Open woodlands and scrub, dry and sunny places. - Uses: None documented.

\section{Elaeagnaceae}

Elaeagnus angustifolia L. - Syn: E. hortensis M. Bieb. - En: Narrow-leaved Oleaster. - Ar: Zayzafoun. - Lf: Tree, 3-5 m. - Ft: April-June. - Mt Hermon: 1197 m, 3329'47'N, 35 50'35'E. Lebanon: Beirut, Tripoli, Bekaa. - Hab: Hedges, old walls, humid soil, low mountain ranges, sunny places. - Uses: Medicinal plant used as antiinflamatory, antipyretic and in kidney disorder. Used also as insect repellent.

\section{Euphorbiaceae}

Chrozophora obliqua (Vahl) Spreng. - Syn: Ch. hierosolymitana Spreng., Ch. verbascifolia (Willd.) Spreng., Croton verbascifolium Willd., C. obliquum Vahl - En: Dyer's Croton Giradol, Turnsole. - Ar: Abad El Shams Lmakdasi. - Lf: Spreading herb, 15-20 cm. - Ft: May-October. - Mt Hermon: 1197 m, 3329'47’N, 35 503'5'E. - Lebanon: Baalbek, Laboue. - Hab: Dry and rocky slopes, gravel soil, roadsides, sunny and ruderal places. - Uses: The juice of fresh leaves and the mixture of the fine powder of dried leaves and oil is used in the treatment of wounds and pimples.

Euphorbia chamaepeplus Boiss. \& Gaill. - Syn: Tithymalus chamaepeplus (Boiss. \& Gaill.) Soják - En: Dwarf Petty Spurge. - Ar: Forfoukh El Ard. - Lf: Semi-shrub, 10-50 cm. - Ft: March-April. Mt Hermon: 1250 m, 33 28'54'N, 35 51'35'E. - Lebanon: Qaa. - Hab: Rocky slopes, mountain pastures, fields in low mountain ranges, dry and sunny places. - Uses: Medicinal plant, fruit is used as antiseptic and anthelmintic. Stem latex is applied on affected area with warts, herpes and dermatitis.

Euphorbia gaillardotii Boiss. \& C. I. Blanche - Syn: Tithymalus gaillardotii (Boiss. \& C. I. Blanche) Klotzsch \& Garcke - En: Gaillardot's spurge. - Ar: Oum El Hobra. - Lf: Semi-shrub, glabrous, up to $60 \mathrm{~cm}$. - Ft: June-Sept. - Mt Hermon: 1553 m, 3327'21' N, 35 52'40”'E. - Lebanon: Majdel Anjar, Baalbek, Ouadi El Harir, Rachaiya. - Hab: Rocky slopes, mountain pastures, fields, dry and sunny places. - Uses: Medicinal plant, fruit is used as anthelmintic.

Euphorbia hierosolymitana Boiss. - Syn: E. thamnoides Boiss. - En: Woody Spurge. - Ar: Halabloub. - Lf: Perennial subshrub, 30-50 cm. - Ft: March-June. - Mt Hermon: $1292 \mathrm{~m}$, $33^{\circ} 28^{\prime} 54^{\prime \prime N}, 35^{\circ} 51^{\prime} 35^{\prime \prime} E$. - Lebanon: Khalde, Jounieh, Nahr El Kalb, Tripoli, Ghazir, Aintoura, 
Ghosta, Mkalles, Kahale, Raifoun, Taanayel, Yammoune. - Hab: Dry scrubs, rocky cliffs at medium altitudes, cultivated and abandoned lands, roadsides, sunny places. - Uses: Latex is externally used to stop bleeding.

Euphorbia macroclada Boiss. - Syn: E. damascena Boiss., E. tinctoria Boiss. \& A. Huet - En: Largebranched Spurge. - Ar: Farbyoun Mashkouk. - Lf: Perennial herb, 30-60 cm. - Ft: April-August. - Mt Hermon: 1191 m, 3328'55'N, 3551'37'E. - Lebanon: Hasroun, Ariz, Ehden, Dimane, Jabal Sannine, Qurnat as Sawda, Zahle, Mreijat, Baalbek, Rachaiya. - Hab: Scrubs, open shrubby places, roadsides, cultivated and abandoned lands, dry and sunny places. - Uses: The stem sap (latex) is applied locally against warts. It is also used as ichthyotoxic and anaesthetic by fishermen.

\section{Fabaceae (Leguminosae)}

Astragalus angustifolius Lam. subsp. angustifolius - Syn: Astragalus hermoneus Boiss.; for taxonomy see Podlech \& Zarre 2013: 2358). - En: Hermon Milk-vetch. - Ar: Kotad Haramon. - Lf: Shrub, 5-15 cm. - Ft: April-August. - Mt Hermon: 2306 m, 33²5’37'N, 3550'32'”E. - Lebanon: Jabal El Barouk, Sannine, Ariz, Marjhine, Jabal El Qamouaa, Marj, Mt Hermon. Endemic to Lebanon and Syria. - Hab: Rocks, crevices, roadsides, high mountain ranges, dry and warm places. - Uses: Ornamental and medicinal plant used as astringent.

Astragalus coluteoides Willd. - Syn: A. damascenus DC. non Boiss. \& Gaill. (see Podlech \& Zarre 2013) - En: Bladder Senna Milk Vetch. - Ar: Kitad Kansouri. - Lf: Dwarf shrub, 10-30 cm. - Ft: May-August. - Mt Hermon: 2060 m, 33 25'57'N, 3550'30'E. - Lebanon: Maasser El Chouf Cedar Forest, Barouk, Hasroun, Berkacha, Ehden, Qurnat as Sawda, Makmel, Yammoune. Endemic to Lebanon and Syria. - Hab: Open and dry places, calcareous rocky slopes, high mountain ranges. Uses: Decoction of roots taken orally to treat diabetes and jaundice.

Astragalus cruentiflorus Boiss. - Syn: Astracantha cruentiflora (Boiss.) Podlech - En: Redflowered Milk-vetch. - Ar: Kotad Ahmar. - Lf: Shrub, 10-30 cm. - Ft: June-August. - Mt Hermon: 1243 m, 33 28'40”N, 35 51'41'E. - Lebanon: Qamouaa, Ehden, Mt Hermon. Endemic to Lebanon and Syria. - Hab: Dry scrubs, among mountain rocks forming large colonies, open shrubby places, low mountain ranges, sunny places. - Uses: Medicinal plant, decoction of roots is orally used to treat diabetes and jaundice. Ornamental.

Astragalus oleaefolius DC. - Syn: Astragalus deinacanthus Boiss., Astracantha deinacantha (Boiss.) Podlech; for taxonomy see Podlech \& Zarre 2013: 1388). - En: Dangerous-spined Milkvetch. - Ar: Kotad Jareh. - Lf: Shrub, 30-50 cm. - Ft: June-Oct. - Mt Hermon: 2307 m, 33²5'39”N, 35 50'31'E. - Lebanon: Sawfar, Dahr Al Baidar, Chtaura, Ksara, Zahle, Hammara, Ouadi El Harir, Baalbek, Rachaiya. - Hab: Rocky calcareous slopes, scrubs, hedges, woodland, field margins, roadsides, dry and sunny places. - Uses: Medicinal and ornamental plant, decoction of roots is orally used as emollient and to treat diabetes and jaundice.

Bituminaria bituminosa (L.) C. H. Stirt. - Syn: Psoralea bituminosa L., Ps. palaestina Gouan, Asphalthium bituminosum (L.) Medik. - En: Bitumen Pea, Pitch Trefoil. - Ar: Homan Homri. - Lf: Perennial herb, 30-60 cm. - Ft: April-July. - Mt Hermon: 1147 m, 33 30'07'N, 3549'41'E. Lebanon: Beirut, Hazmiyeh, Antilias, Tripoli, Aley, Moukhtara, Bcharre, Ksara, Taanayel, Zahle, Sarada. - Hab: Open shrubby areas, vineyards, pastures, meadows, sunny places. - Uses: Medicinal plant, leaves and fruits are used in the treatment of intestinal ailments and gastric ulcers. 
Coronilla scorpioides (L.) W. D. J. Koch - En: Annual Scorpion-vetch. - Ar: Ekleel El Akrab. - Lf: Annual plant, 10-40 cm. - Ft: Feb-April. - Mt Hermon: 1201 m, 33²9'14’N, 3551'10'E. Lebanon: Beirut, Beirut River, Nahr El Kalb, Tripoli, Broumana, Aabey, Aintoura, Sarada, Sannine, Hasroun, Rayak, Zahle, Maalaqa, Rachaiya. - Hab: Cultivated and abandoned lands, roadsides, open shrubby areas, dry and sunny places. - Uses: None documented.

Medicago sativa L. - En: Lucerne, Alfalfa. - Ar: Fossa Molawana. - Lf: Perennial herb, $30-80 \mathrm{~cm}$. - Ft: All year. - Mt Hermon: 1426 m, 3337'20’N, 35 50'37’E. - Lebanon: Tripoli, Bikfayia, Ariz, Hadath, Dimane, Zahle, Baalbek, Jabal El Knisse. - Hab: Roadsides, fields, clay soils, sunny places. - Uses: Medicinal plant, flowering aerial part is used as diuretic, anti-inflammatory, anti-hemorrhagic, anticholesteremic, and as a good source of phytoestrogen.

Ononis spinosa subsp. leiosperma (Boiss.) Širj. - Syn: O. leiosperma Boiss., O. repens subsp. leiosperma (Boiss.) Greuter - En: Spiny Restharrow. - Ar: Shobrok Shaaek. - Lf: Dwarf shrub, 30-50 cm. - Ft: April-August. - Mt Hermon: 1250 m, 33²8'54’N, 3551'35”'E. - Lebanon: Saida, Beirut, Bikfayia, Ghazir, Moukhtara, Dimane, Hasroun, Ariz, Zahle, Taanayel, Ouadi El Harir. - Hab: Dry and rocky slopes, roadsides, fields, sunny places. - Uses: Medicinal plant, decoction of flowering aerial parts is used as antiseptic in eczema and the treatment of kidney stones and other urinary tract disorders.

Ononis talaverae Devesa \& G. López - Syn: $O$. natrix subsp. natrix sensu Förther \& Podlech non L.; O. natrix, as given for Lebanon by Förther \& Podlech (1991: 269), is in fact a W Mediterranean element confined to SW Europe (Devesa \& López 1997). - En: Shrubby Rest Harrow. - Ar: Lezzayk, Shobrok Thobani. - Lf: Perennial subshrub, 50-100 cm. - Ft: March-December. - Mt Hermon: 1230

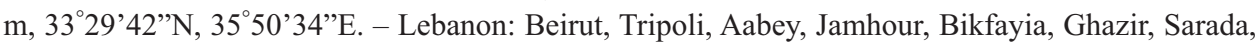
Ehden, Qannoubine, Hadath, Ain Zhalta, Bcharre, Ksara, Masnaa. - Hab: Open and dry places,cultivated and abandoned lands. - Uses: or Decoction or infusion of flowering stems is externally applied as antiseptic for eczema and is orally used for kidney stones and disorders of the urinary tract. Root decoction used orally as diuretic.

Scorpiurus muricatus L. - Syn: S. sulcatus L., S. subvillosus L. - En: Furrowed Caterpillar. - Ar: Akrabeyyi. - Lf: Annual herb, 10-40 cm. - Ft: April-May. - Mt Hermon: 1140 m, 33 30'07'N, 3549'41'E. - Lebanon: Saida, Beirut, Jamhour, Mkalles, Qoub Elias, Rayak, Ebel Es Saqi. - Hab: Meadows, fields, roadsides, abandoned lands, dry and sunny places. - Uses: Edible plant (raw in salads) and medicinally used for scorpion stings.

Trifolium pilulare Boiss. - En: Ball Cotton Clover. - Ar: Nefl Hebani. - Lf: Annual herb, 6-25 cm. - Ft: March-May. - Mt Hermon: 1191 m, 3328'55'N, 3551'37'E. - Lebanon: Beirut, Tripoli, Ehmej, Beit Meri, Bikfayia, Terbol, Broumana, Aabey, Raifoun, Mdeyrej, Dahr Al Baidar, Niha, Zahle, Wadi El Harir, Rachaiya. - Hab: Grassy grounds, scrubs, cultivated and abandoned lands, dry open woodlands, roadsides. - Uses: Decoction of leaves is locally applied as vulnerary.

Trifolium purpureum Loisel. - Syn: T. desvauxii Boiss. \& C. I. Blanche, T. purpureum var. desvauxii (Boiss. \& C. I. Blanche) Post, T. purpureum var. roussaeanum Holmboe non T. roussaeanum Boiss. - En: Purple Clover. - Ar: Nefl Orjouani. - Lf: Annual herb, 20-50 cm. - Ft: March-June. Mt Hermon: 1191 m, 33²8'55’N, 3551'73’E. - Lebanon: Ras El Biyada, Tyr, Saida, Ras Jedra, Bir Hassan, Damour, Beirut, Antilias, Nahr El Kalb, Tripoli, Broumana, Baabda, Baskinta, Sfire, Mdeyrej, Baalbek, Ain Aata. - Hab: Grassy grounds, scrubs, cultivated and abandoned lands, dry open woodlands, roadsides. - Uses: Decoction of plant is locally used to treat rheumatism. 
Trifolium tomentosum L. - Syn: Trifolium curvisepalum Täckh. - En: Tomentose Clover. - Ar: Nfel Mshaar. - Lf: Perennial herb, 10-40 cm. - Ft: Feb-May. - Mt Hermon: 1140 m, 3330'07'N, 35 49'41'E. - Lebanon: Beirut, Beit Meri, Qartaba, Hasbaya, Aabey, Nahr El Damour, Bikfayia, Kfar Matta, Zahle, Tripoli, Rachaiya. - Hab: Dry grassy habitats, abandoned lands, roadsides, sunny places. - Uses: Medicinal plant, root decoction is diuretic and used against vomiting, fever and cough.

Vicia tenuifolia Roth - Syn: Vicia cracca subsp. tenuifolia (Roth) Bonnier \& Layens, Vicia boissieri Freyn - En: Bramble vetch, Tare. - Ar: Baykeyya Dayyekat El Warak. - Lf: Perennial climbing herb, 1-2 m. - Ft: May-July. - Mt Hermon: 1220 m, 3328'31'N, 3551'10”E. - Lebanon: Ehden, Faraiya, Akkar, Ariz, Bcharre, Jabal Barouk, Sannine, Bhamdoun, Jabal El Knisse, Mt Hermon. - Hab: Cultivated lands, damp meadows, hedges, fields. - Uses: Melliferous, forage plant.

\section{Fagaceae}

Quercus coccifera L. - Syn: Q. calliprinos Webb, Q. coccifera var. calliprinos (Webb) Boiss. - En: Palestine Oak, Kermes Oak. - Ar: Ballout Sendian. - Lf: Evergreen tree, 2-20 m, trunk diameter up to $1 \mathrm{~m}$. The habit whether shrubby ("coccifera"-type) or tree-like ("calliprinos"- type) is of no taxonomic value. - Ft: February-April. - Mt Hermon: 1766 m, 3326’35”N, 3550'44”E. - Lebanon: Beirut, Nahr Ibrahim, Madfoun, Nahr Abou Ali, Ain Trass, Jezzine, Aabey, Jamhour, Beit Meri, Bikfayia, Baabda, Qraiaa, Salima, Douma, above Maasser El Chouf Cedar Forest, Ghazir, Ehden, Wadi Qannoubine, Dimane, Hasroun, Hadchit, Ouadi El Harir, Ammiq, Deir El Ahmar. - Hab: Open rocky slopes in high mountain ranges, forming large colonies. - Uses: Decoction of bark is astringent and used to treat wounds and sores.

\section{Geraniaceae}

Geranium libanoticum Schenk - En: Lebanese Geranium. - Ar: Ghurnuqi Lubnani. - Lf: Perennial herb, tuberous geophyte, 20 cm. - Ft: April-May. - Mt Hermon: 1255 m, 3329'08'N, 35 51'29'E. - Lebanon: Ariz, Faraiya, Maasser. - Hab: Rocky slopes, fields, red clay soil (terra rossa), sunny places. - Uses: None documented.

Geranium tuberosum L. - En: Bulbous crane's-bill, Tuberous Geranium. - Ar: Gharnook Askouli. - Lf : Perennial herb, tuberous geophyte, 10-40 cm. - Ft: March-June. - Mt Hermon: $1140 \mathrm{~m}$, 33 30'07'N, 35 49'41'E. - Lebanon: Saida, Beirut, Nahr El Kaleb, Nahr Ibrahim, Tripoli, Asfouriye, Aabey, Chemlan, Jounieh, Hasroun, Ariz, Ehden, Ainata, Tanaayel, Zahle, Rayak, Akkar, Sarada. - Hab: Cultivated lands, roadsides, rocky slopes in the mountains. - Uses: Decoction of plant is externally used in the treatment of hemorrhoids.

\section{Grossulariaceae}

Ribes orientale Desf. - En: Oriental Currant. - Ar: Kishmish Sharqi. - Lf: Shrub, 50-100 cm. - Ft: May-June. - Mt Hermon: 1802 m, 3326’31'N, 3550'49'E. - Lebanon: Aaqoura, Yammoune, Ariz, Ariz El Hadath, Danniyeh, Mt Hermon. - Hab: Rocky slopes, high mountain ranges. - Uses: Edible plant, fruit is eaten raw or cooked and used as a purgative.

\section{Iridaceae}

Crocus aleppicus Baker - Syn: C. hyemalis var. gaillardotii Boiss. \& C. I. Blanche, C. gaillardotii (Boiss. \& C. I. Blanche) Maw - En: Aleppo Crocus. - Ar: Hanin, Zaafaran Halab. - Lf: Herbaceous 
geophyte, 4-8 cm. - Ft: November-January. - Mt Hermon: 1201m, 33²9'20”N, 3551'00'E. Lebanon: Bourj El Brajne, Beirut, Chekka, Zgharta, Tripoli, Ammiq, Kefraiya, Baalbek, Ouadi El Harir, Joub Jannine, Yanta and Rachaiya. - Hab: Damp stony lands, among rocks, open shrubby areas, scrubs, roadsides. - Uses: Corms and soft seeds are edible raw or roasted. Flower stigmas are narcotic for asthma and cough.

Gladiolus atroviolaceus Boiss. - Syn : Gladiolus aleppicus Boiss. - En: Aleppo Gladiolus. - Ar: Dalbouth Halab. - Lf: Perennial plant, bulbous, 30-100 cm. - Ft: March-May. - Mt Hermon: 1228 m, 33 $28^{\prime} 44^{\prime \prime N}, 35^{\circ} 51^{\prime} 12^{\prime \prime} E$. - Lebanon: Chtaura, Zahle, Masnaa, Soultane Yaaqoub, Hasbaya, Rachaiya. - Hab: Cultivated land (particularly wheat fields), roadsides, hill slopes, dry and sunny places. - Uses: Medicinal and ornamental plant, decoction of roots is used to relieve stomachache. Ornamental.

Iris histrio Rchb. f. - Syn: I. libani Baker - En: Histrio Iris. - Ar: Sawsan Mowachah. - Lf: Herbaceous geophyte, 15-25 cm. - Ft: February-March. - Mt Hermon: 1677 m, 3326'41'N, 35 50'32'E. - Lebanon: Saida, Ghazir, Sawfar, Bhamdoun, Mdeyrej, Dahr Al Baidar, Jounieh, Qadisha valley, Marjayoun, Ain Lejgi towards Ariz El Barouk, Jezzine, Zahle. - Hab: Among mountain rocks, damp places at medium altitudes. - Uses: Decoction of rhizomes is used as diuretic in hydropsy. Ornamental.

\section{Ixioliriaceae}

Ixiolirion tataricum (Pall.) Schult. \& Schult. f. - Syn: I. montanum (Labill.) Schult. \& Schult. f., I. pallasii Ledeb., Amaryllis tatarica Pall., A. montana Labill. - En: Siberian Lily, Lavender Mountain Lily. - Ar: Khouzama Al Jabal, Sawsan Al Jabal. - Lf: Perennial herb, 40-50 cm. - Ft: April-May. Mt Hermon: 2040 m, 33²8'58”N, 35 50'35”E. - Lebanon: Bhamdoun, Maasser El Chouf, Jouar Bakich, Jezzine, Machgara, Akkar, Ehden, Tannourine, Hasroun, Ariz, Dahr Al Baidar, Jabal El Barouk, Zahle, Sannine, Rachaiya. - Hab: Steep rocky slopes and cliffs in hills and mountains, sunny places. - Uses: Ornamental.

\section{Lamiaceae (Labiatae)}

Ajuga chamaepitys subsp. palaestina (Boiss.) Bornm. - Syn: A. palaestina Boiss., A. chia var. suffrutescens Boiss., A. chia var. tridactylites Boiss. non A. tridactylites Benth. - En: Three-fingered Bugle. - Ar: Messayki Falastinie. - Lf: Annual herb. - Ft: May-November. - Mt Hermon: 1267 m,

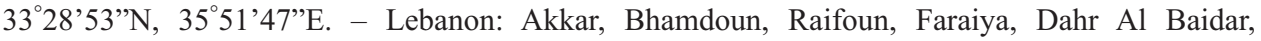
Berkacha, Qannoubine, Bekacha, Ariz, Hasroun, Jabal El Knisse, Ehden, Machghara, Hermel, Baalbek, Joub Jannine, Mt Hermon. - Hab: Rock-crevices, gravel soil, rubble by roadsides, woodlands, barren, sunny places. - Uses: Dried powdered plant or its infusion is taken after meals in diabetes and infusion of aerial parts is used as antidiarrheic.

Ajuga orientalis L. - En: Oriental Bugle. - Ar: Orsof Sharki. - Lf: Perennial plant, non-woody, 30$50 \mathrm{~cm}$. - Ft: April-June. - Mt Hermon: $1201 \mathrm{~m}, 33^{\circ} 28^{\prime} 40^{\prime \prime} \mathrm{N}, 35^{\circ} 51^{\prime} 12^{\prime \prime} \mathrm{E}$. - Lebanon: Batroun, Ras Chekka, Tripoli, Saida, Deir Al Qamar, Ghazir, Aramoun, Jezzine, Ghosta, Ras El Metn, Machgara, Mdeyrej, Chtaura, Qoub Elias, Zahle, Taanayel, Rachaiya, Ramiye, Baalbek. Hab: Roadsides, growing through cracks in concrete, gardens, fields. - Uses: Medicinal plant, decoction of aerial parts is used in the treatment of diabetes, gastritis, diarrhea, ulcer, and stomachache.

Ballota saxatilis C. Presl - Syn: B. rugosa (Desf.) Benth., B. rugosa (Banks \& Solander) Dinsm. - 
En: Rock Horehound. - Ar: Asfan. - Lf: Perennial herb, 20-60 cm. - Ft: May-September. - Mt

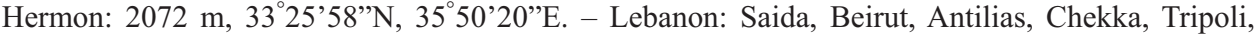
Terbol, Douma, Ghazir, Machghara, Hasroun, Barouk, Zahle, Rachaiya and Hasbaya. - Hab: Open shrubby areas, rocky slopes, low and middle mountain ranges, roadsides, dry and sunny places. Uses: Infusion of flowering top is used as antispasmodic and vermifuge.

Lamium amplexicaule L. - En: Henbit Deadnettle, Greater Henbit. - Ar: Ras El Her. - Lf: Annual herb, 10-30 cm. - Ft: Nearly all year. - Mt Hermon: 1159 m, 33³0'06”N, 3549'52”E. - Lebanon: Saida, Beirut, Tripoli, Qraiaa, Qoubaiyat, Hasroun, Zahle, Ain Zhalta, Barouk, Sarada. - Hab: Cultivated and abandoned lands, vineyards, olive groves, meadows, low mountain ranges, sunny places. - Uses: Melliferous.

Marrubium globosum subsp. libanoticum (Boiss.) P. H. Davis - Syn: M. hermonis Boiss., M. libanoticum Boiss. var. hermonis (Boiss.) Boiss. - En: Lebanon White Horehound. - Ar: Al Ghabra, Frasyoun Lebnan. - Lf: Perennial subshrub, 15-40 cm. - Ft: June-October. - Mt Hermon: 2345 m, $33^{\circ} 25^{\prime} 35^{\prime}$ 'N, 35 50'40'E. - Lebanon: Jabal Sannine, Ehmej, Ariz, Qornat as Sawda. - Hab: Rocky slopes, stony and grassy grounds, calcareous soil, roadsides, medium and high mountain ranges. Uses: Infusion of leaves is orally used in the treatment of jaundices, fever and stomachache.

Mentha spicata subsp. condensata (Briq.) Greuter \& Burdet - Syn: M. sylvestris L. var. stenostachya Boiss., M. canescens Sieber non Roth, M. sieberi K. Koch, M. microphylla K. Koch - En: Spearmint, Spear Mint. - Ar: Naanaa Barri. - Lf: Perennial herb, aromatic, 50-100 cm. - Ft: JuneDecember. - Mt Hermon: 2417 m, 3325'35'N, 35 50'40”E. - Lebanon: Tyre, Beirut, Antelias, Nahr El Kalb, Nahr Ibrahim, Tripoli, Aabey, Raifoun, Kahale, Hasroun, Knisse, Qamoua, Akoura, Tannourine, Ehden, Ariz, Niha, Zahle, Chtaura, Taanayel, Nabee El Asal, Yammoune, Mt Hermon. - Hab: Rocky slopes, damp habitats, streams, ditches, cultivated lands, high mountain ranges, sunny places. - Uses: Infusion of leaves and flowering summits is used as carminative, stomachic and for abdominal pains. Leaves infusion is taken as tea against headache and colds.

Micromeria myrtifolia Boiss. \& Hohen. - Syn: M. juliana var. myrtifolia (Boiss. \& Hohen.) Nyman, Satureja myrtifolia (Boiss. \& Hohen.) Greuter \& Burdet - En: Myrtle-leaved Savory. - Ar: Zoufa. - Lf: Dwarf perennial subshrub, 20-60 cm. - Ft: April-September. - Mt Hermon: 1191 m, 33²8'55'N, 35 51'37'E. - Lebanon: Saida, Beirut, Tripoli, Nahr El Kalb, Kahale, Aabey, Douma, Deir Al Qamar, Bzemmar, Bhamdoun, Aachqout, Raifoun, Broumana, Rachaiya, Deir El Ahmar, Jdita, Yammoune. Hab: Rocky hillslopes, scrubs, open pine forests, calcareous plateaus, low mountain ranges, sunny places. - Uses: Infusion of aerial parts is used as febrifuge and to relieve colds and coughs.

Moluccella laevis L. - En: Bells of Ireland. - Ar: Ajras Bayt Lahm. - Lf: Annual plant, 50-100 cm. - Ft: June-July. - Mt Hermon: 1195 m, 3329'54’N, 35 49’49”E. - Lebanon: Bhamdoun, Sawfar, Hermel, Mchaitie, Taanayel, Haouch Al Oumara, Joub Jannine, Zahle, Ouadi El Harir, Rachaiya. Hab: Fields, sunny places. - Uses: Ornamental plant.

Nepeta cilicica Benth. - En: Cilician Catmint. - Ar: Kotrum kilikia. - Lf: Annual herb, aromatic, 30-80 cm. - Ft: June-September. - Mt Hermon: 2033 m, 3325'58’N, 35 50’35’E. - Lebanon: Ain Zhalta, Maasser El Chouf, Arz El Barouk, Knisse, Sannine, Yammoune, Bcharre, Ehden, Qurnat as Sawda, Rachaiya. - Hab: Dry gravel soil, among rocks in high mountain ranges, sunny places. Uses: Infusion of the aerial part is traditionally used as carminative and in the treatment of nervous disorders. Oils of aerial parts and seeds are used in the treatment of rheumatism. Flowers and seeds are used as antiseptic and antispasmodic and in the treatment of cold, toothache and dysentery. 
Nepeta curviflora Boiss. - En: Syrian catmint. - Ar: Qatram muqawwas al zahr. - Lf: Perennial herb. - Ft: April-July. - Mt Hermon: 1195 m, 33²9’54’N, 3549’49’E. - Lebanon: Saida, Rmeile, Antilias, Qalmoun, Tripoli, Araya, Annaya, Ghazir, Ghosta, Ghine, Tourzaya, Mairouba, Majdel Balhis, Deir El Qamar, Mdeyrej, Ehden, Ain Zhalta, Chtaura, Ksara. - Hab: Dry rocky places, calcareous screes, roadside banks. - Uses: Condiment and medicinal plant used to cure digestive disorders and respiratory diseases.

Nepeta glomerata Benth. - En: Glomerate Catmint. - Ar: Kotrum Moutajamme. - Lf: Perennial herb, 10-50 cm. - Ft: June-September. - Mt Hermon: 2400 m, 33 25'33'N, 35 50'48'E. - Lebanon: Sannine, Makmel, Ariz, Qurnat as Sawda, Ainata, Mt Hermon. - Hab: Dry gravel soil, among rocks in high mountain ranges, sunny places. - Uses: Infusion of the aerial part is traditionally used as carminative and in the treatment of nervous disorders. Seed oils is locally applied in the treatment of rheumatism and infusion of aerial parts is orally taken against dysentery.

Nepeta italica L. subsp. italica - Syn: Glechoma italica (L.) Kuntze - En: White-covered Catmint. - Ar: Kotrom Abyad. - Lf: Perennial herb, 10-40 cm. - Ft: May-August. - Mt Hermon: 1243 m, $33^{\circ} 28^{\prime} 40^{\prime \prime N}, 35^{\circ} 51^{\prime} 41^{\prime \prime} E ., 1481 \mathrm{~m}, 33^{\circ} 28^{\prime} 41^{\prime \prime N}, 35^{\circ} 50^{\prime} 27^{\prime \prime E}$. - Lebanon: Jebaa, Laqlouq, Maasser El Chouf, Aaqoura, Bhamdoun, Jezzine, Ain Zhalta, Mairouba, Tartij, Faraiya, Barouk, Ouadi El Harir, Mdeyrej, Jabal El Knisse, Afqa, Sannine, Ariz, Ehden, Bcharre, Hadath, Hadchit, Jabal El Qamouaa, Yammoune, Deir El Ahmar, Ain Hazir, Ainata, Baalbek, Nabi Chite, Yanta, Rachaiya. Hab: Rocky slopes, red soil, medium mountain ranges, limestone gravel soil, sunny places. - Uses: Infusion of the aerial part is orally used as carminative and in the treatment of nervous disorders. Oils of aerial parts and seeds are used for treatment of rheumatism. Flowers are edible and melliferous.

Phlomis brevilabris Boiss. - En: Short-lipped Phlomis. - Ar: Odaynat Meeza. - Lf: Perennial subshrub, 20-60 cm. - Ft: May-August. - Mt Hermon: 1843 m, 33²6'24'N, 35 55'44'E. - Lebanon: Jabal El Barouk, Ariz, Ain Zhalta, Jabal El Knisse, Sannine, Jisr Al Hajar, Akoura, Dimane, Saghbine, Ehden, Hermel, Hasroun, Chebaa. Endemic to Lebanon and Syria. - Hab: Calcareous rocky plateaus, medium and high mountain rages. - Uses: None documented.

Phlomis chrysophylla Boiss. - En: Golden-leaved Phlomis. - Ar: Odaina Dahabiyya. - Lf: Perennial subshrub, 30-100 cm. - Ft: May-August. - Mt Hermon: 1325 m, 33²8'27'N, 35503'9'E. Lebanon: Ain Zhalta, Mdeyrej, Jabal El Knisse, Faraiya, Afqa, Jabal Qamoua, Douma, Ehden, Hasroun, Bcharre, Ariz, Ainata, Qalaat El Chekif, Hasbaya. - Hab: Calcareous rocky plateaus, medium and high mountain ranges. - Uses: Infusion of flowering parts is orally taken to treat cough.

Salvia indica L. - Syn: S. brachycalyx Boiss. - En: Blue Sage. - Ar: Kaseen Hindi, Mayramyie Zarkaa. - Lf: Annual herb, 80-150 cm. - Ft: April-July. - Mt Hermon: 1283 m, 3328'26”N, 35 50'49'E. - Lebanon: Dahr Al Baidar, Jabal El Knisse, Zahle, Kefraiya. - Hab: Dry gravel soil, among mountain rocks, low mountain ranges, sunny places. - Uses: Infusion of leaves is used in the treatment of rheumatic pains, and colds.

Salvia microstegia Boiss. \& Balansa - Syn: S. verbascifolia M. Bieb. var. cana Boiss. - En: Fleecy Sage. - Ar: Kouaisa Saghira. - Lf: Perennial herb or subshrub, 40-100 cm. - Ft: June-September. Mt Hermon: 1602 m, 3327’20’N, 3552'45”E. - Lebanon: Jabal El Barouk, Sawfar, Knisse, Sannine, Hasroun, Bcharre, Ariz, Ehden, Mt Hermon. - Hab: Dry gravel soil, among mountain rocks, medium mountain ranges, sunny places. - Uses: Infusion of leaves and summits is used as antiseptic, sudorific, antispasmodic and to relieve cold. 
Salvia multicaulis Vahl - En: Pinard's Sage. - Ar: Saq Al Arus. - Lf: Perennial herb, 30-50 cm. Ft: April-June. - Mt Hermon: 1224 m, 33²9’08’N, 3551'29”E. - Lebanon: Zahle, Ouadi El Harir. - Hab: fields, roadsides. - Uses: Medicinal plant used as anti inflammatory, analgesic and treatment of stomach disturbances. Used also as condiment for tea.

Salvia rubifolia Boiss. - En: Bramble-leaved Sage. - Ar: Kouaisa Olaykiat Al Warak. - Lf:

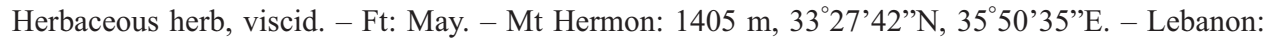
Ainata, Kefraiya, Aitanit, Ouadi El Harir, Masnaa, Ouadi Yahfoufa, Rachaiya. Endemic to Lebanon and Syria. - Hab: Dry gravel soil, among mountain rocks, medium mountain ranges, sunny places. - Uses: Infusion of leaves and young shoots is orally used for cough, influenza, cold and locally as vulnerary.

Scutellaria utriculata Labill. - En: Bladder Skullcap. - Ar: Harboon Korbi. - Lf: Perennial herb,

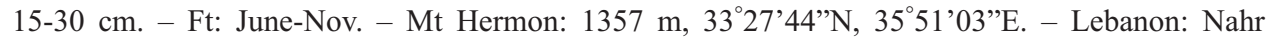
Ibrahim, Amchit, Bhamdoun, Ain Zhalta, Ehden, Danniyeh, Hadath, Tannourine, Jabal El Barouk, Jabal El Knisse, Ain Hazir, Ariz, Ouadi El Arayech, Ainata, Mreijat, Zahle, Yanta, Baalbek, Mt Hermon area. Endemic to Lebanon and Syria. - Hab: Scrubs, open shrubby areas, calcareous cliffs, crevices, hedges, medium mountain ranges, rocky and sunny places. - Uses: Medicinal plant, infusion of aerial parts is used as diuretic and sedative for neurological disorders and the treatment of digestion problems. Ornamental.

Sideritis libanotica Labill. - En: Lebanon Iron-wort. - Ar: Fokkah Lebnani. - Lf: Perennial herb. Ft: July-August. - Mt Hermon 1843 m, 3326'24’N, 3555'44’'E. - Lebanon: Jabal El Barouk, Sawfar, Jabal El Knisse, Hadath, Akoura, Jabal Sannine, Dimene, Yammoune, Hasroun, Ariz. - Hab: Stony and rocky slopes, scrubs, cultivated and abandoned lands, dry and sunny places. - Uses: None documented.

Stachys libanotica Benth. - En: Lebanon Wound-wort. - Ar: Kartoum Lebnani. - Lf: Perennial herb, 50-100 cm. - Ft: May-July. - Mt Hermon: 1292 m, 33 28'54’N, 35 513'5’'E. - Lebanon: Jabal Niha, Jabal El Knisse, Jabal Sannine, Tannourine, Hazerta, Ouadi El Harir, Rachaiya. - Hab: Scrubs, red gravel soil, low and medium mountain ranges, dry and sunny places. - Uses: Infusion of entire plant is used as anticatarrhal while roots are purgative.

Teucrium capitatum L. subsp. capitatum - Syn: T. polium auct. non L., T. virescens auct. non Pomel, T. polium subsp. album (Garsault) Breistr. - En: Felty Germander, Poley. - Ar: Jaade Tobeyyeh. - Lf: Perennial subshrub, 50-80 cm. - Ft: May-September. - Mt Hermon: $1447 \mathrm{~m}$, $33^{\circ} 27^{\prime 2} 21^{\prime} N$, 35 51'46”'E. - Lebanon: Beirut, Maameltein, Saida, Jamhour, Kahale, Ghemlan, Zahrani, Saidit El Mantara, Bikfayia, Akoura, Jabal El Knisse, Jabal El Barouk, Zahle, Ain Zhalta, Dahr Al Baidar, Mar Semaane, Qaraoun, North of Qaa, Rachaiya. - Hab: Scrubs, mountain slopes, from sealevel to the high summits of Lebanon. - Uses: Widely used as sedative and antidiabetic.

Teucrium orientale L. subsp. orientale - Syn: T. nivale Boiss. - En: Oriental Germander, Greatflowered Germander. - Ar: Jaada Sharkiyya. - Lf: Perennial subshrub, 20-150 cm. - Ft: May-July. Mt Hermon: 1843 m, 33²6'24’N, 35 55'44'E. - Lebanon: Ehden, Hasroun, Laqlouq, Jabal El Knisse, Ariz, Douma, Aaqoura, Qaa El Rim, Mt Hermon. - Hab: Dry rocky slopes, abandoned lands, high mountain ranges. - Uses: Infusion of aerial parts is used as astringent, vulnerary and febrifuge.

Teucrium pruinosum Boiss. - En: Pruinose Germander. - Ar: Maleeh. - Lf: Perennial subshrub, 3050 cm. - Ft: May-July. - Mt Hermon: 2150 m, 33²5'42’N, 35 50'17'E. - Lebanon: Baalbek, 
Hermel. - Hab: Rocky slopes at high altitudes in sub-arid regions, roadsides. - Uses: Hot infusion is used as vulnerary and for stomach and intestinal disorders. Plant is used in a steam bath to relieve colds and fevers.

Ziziphora clinopodioides Lam. subsp. clinopodioides - Syn: Z. canescens Benth., Z. clinopodioides var. canescens (Benth.) Boiss. - En: Hoary Ziziphora. - Ar: Innabiya Baydaa. - Lf: Annual herb, 10-

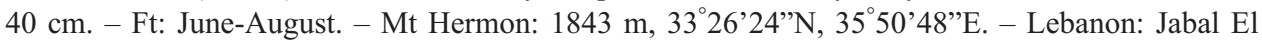
Barouk, Jabal El Knisse, Jabal Sannine, Dimane, Bcharre, Ariz, Makmel, Qurnat as Sawda. - Hab: Mountain rocks, calcareous plateaus, rocky hillslopes athigh altitudes, sunny places. - Uses: Infusion of aerial parts is internally used to treat colds, coughs, stomachache and relieve pains.

\section{Liliaceae (s. str.)}

Gagea fragifera Ehr. Bayer \& G. López - Syn: G. anisanthos K. Koch, G. liotardii (Sternb.) Schult. \& Schult. f. - En: Unequal Flowered Gagea. - Ar: Ghajiya Moutabaynat Al Zahr. - Lf: Perennial herb, bulbous geophyte, 10-20 cm. - Ft: April-June. - Mt Hermon: $1157 \mathrm{~m}$, $33^{\circ} 30^{\prime} 05^{\prime \prime} N$, 3549'49”E. - Lebanon: Jabal El Barouk, Jabal El Knisse, Laqlouq, Jabal Sannine, Tannourine, Hasroun, Ariz. - Hab: Dry hillslopes and stony places, scrub, grassy grounds. Uses: None documented.

\section{Linaceae}

Linum pubescens Banks \& Sol. - En: Downy Flax, Pink Flax. - Ar: Kottan Aazghab. - Lf: Annual herb, 30-80 cm. - Ft: March-May. - Mt Hermon: 1147 m, 33 30’07’'N, 35 49’41'E. - Lebanon: Tyre, Aadloun, Saida, Beirut, Ras Chekka, Tripoli, Akkar, Maghdouche, Aabey, Maasser, Jamhour, Bsous, Choueifat, Terbol, Deir Al Qamar, Raifoun, Mreijat, Akkar, Zahle, Mt Hermon area. - Hab: Rocky slopes, calcareous cliffs, roadsides, forest margins. - Uses: Medicinal plant, decoction of aerial parts and flowers are used in the treatment of diabetes, cancer, for weight loss and to relieve headache.

\section{Loranthaceae}

Viscum album L. subsp. album - En: White Mistletoe. - Ar: Hadal Aabyad. - Lf: Shrub, woody at the base, 20-60 cm. - Ft: March-April. - Mt Hermon: 1481 m, 3328'41'N, 35 50'27'E. - Lebanon: Yammoune, Marj, Ammiq, Baalbeck, abundant in Rachaiya. - Hab: Parasitic on fruit trees of Rosaceae. - Uses: Medicinal plant used for respiratory disorders.

\section{Malvaceae}

Alcea acaulis (Cav.) Alef. subsp. acaulis - Syn: Althaea acaulis Cav. - En: Stemless Hollyhock. Ar: Khtmyia Doun Sak. - Lf: Perennial herb, 5-10 cm. - Ft: April-May. - Mt Hermon: 1200 m, $33^{\circ} 29^{\prime} 16^{\prime} \mathrm{N}, 35^{\circ} 51^{\prime} 08^{\prime}$ E. - Lebanon: Tripoli, Jabal Terbol, Ehden, Ariz, Decline of Makmel, Saadnayel, Baalbek, Chlifa, Hasbaya. - Hab: Sub-mountain and lowland zones on arable land, roadsides and hedges. - Uses: Infusion or decoction of flowers is used as emolient to treat chronic cough, bronchial and throat catarrh.

Alcea damascena (Mouterde) Mouterde - Syn: Althaea damascena Mouterde - En: Damascus Hollyhock. - Ar: Khtmyia Demashk. - Lf: Perennial herb, up to above 3 m. - Ft: May-Sept. - Mt Hermon: 1142 m, 3330'07’N, 3549'41'E. - Lebanon: Fouara, Baalbek, Ras Baalbek, Baalbek. Endemic to Lebanon and Syria. - Hab: Dry scrubs, roadsides, rocky cliffs, sunny places. - Uses: 
Medicinal plant, decoction of flowers is used as emollient (mucilage), diuretic, analgesic, antitussive, anti-inflammatory and in the treatment of cold and flu.

\section{Oleaceae}

Jasminum fruticans L. - Syn: J. syriacum Boiss. \& Gaill. - En: Bush Jasmine. - Ar: Yasmeen Barri. - Lf: Evergreen shrub, 50-150 cm. - Ft: March-May. - Mt Hermon: 1191 m, 33²8'55"N, 35 51'37'E. - Lebanon: Beirut, Antilias, Douma, Deir El Ahmar, Qraiaa, Nabi Chit, Saraain, Hazerta, Ouadi El Harir, Rachaiya. - Hab: Rocky soil. - Uses: Decoction of stems is traditionally used as anthelmintic. Infusion of flowers is used in the form of inhalations for $10 \min 3$ times a day against allergy and asthenia.

\section{Onagraceae}

Epilobium hirsutum L. - En: Hirsute Willow-herb. - Ar: Okays. - Lf: Annual plant, 1 m. - Ft: JuneAugust. - Mt Hermon: 853 m, 35²8'50”N, 3547’38”E. - Lebanon: Saida, Nahr El Kalb, Qraiaa, Salima, Ghazir, Bikfayia, Broumana, Moukhtara, Tannourine, Mazraat Kfar Dibian, Dimane, Hasroun, Bcharre, Jabal Qamouaa, Taanayel, Zahle, Ksara, Bar Elias. - Hab: Banks of streams, fields, open shrubby areas, roadsides, low mountain ranges, damp and sunny places. - Uses: Edible plant and medicinal plant. Decoction of leaves is administered in gastritis, stomach disease, ulceration, inflammation, prostate tumors, rectal bleeding, constipation, menstrual and gastrointestinal disorders.

\section{Orchidaceae}

Himanthoglossum affine (Boiss.) Schltr. - Syn: Aceras affine Boiss., Himantoglossum hircinum subsp. affine (Boiss.) H. Sund., Himantoglossum hircinum var. affine (Boiss.) J. J. Wood, Loroglossum affine (Boiss.) E. G. Camus \& Bergon - En: Related Lizard Orchid. - Ar: Himantoghlosom Al Tays. - Lf: Geophytic herb, $40 \mathrm{~cm}$. - Ft: May-June. - Mt Hermon: $1163 \mathrm{~m}$, $33^{\circ} 29^{\prime} 20^{\prime}$ 'N $35^{\circ} 51^{\prime} 03$ ”'E. - Lebanon: Maasser El Chouf, Khraibe, Beirut. - Hab: Dry and stony hillslopes, half-shady places in sparse deciduous or pine woodland. - Uses: Ornamental plant.

Orchis anatolica Boiss. - En: Anatolian Orchid. - Ar: Orkid Al Anadol. - Lf: Perennial herb, tuberous geophyte 15-40 cm. - Ft: March-April. - Mt Hermon: 1168 m, 33 30'06”N, 3549'52”E. Lebanon: Beirut, Nahr El Kalb, Aabey, Beit Meri, Ghazir, Bikfayia, Souk El Ghareb, Broumana, Jezzine, Machghara, Ehden, Hasroun, Hadath, Ehden, Zahle, Rachaiya. - Hab: Grassy and rocky grounds, scrubs, hillslopes. - Uses: Powdered tuber "Salep" (Sahlab) mixed with hot milk is taken to increase potency.

\section{Papaveraceae (incl. Fumariaceae)}

Fumaria asepala Boiss. - En: White Fumitory. - Ar: Shahatrej Abyad. - Lf: Annual, spreading herb, 10-60 cm. - Ft: March-June. - Mt Hermon: 1140 m, 33³0'07'N, 3549'41'E. - Lebanon: Hadath, Bcharre, Hasroun, Ariz, Yammoune, Baalbek, Rachaiya. - Hab: Stony and rocky slopes, cultivated and abandoned lands, low mountain ranges, sunny places. - Uses: Decoction of flowering tops is orally used as laxative and externally applied as antipruriginous and astringent to cure dermatitis, eczema and scabies. 
Glaucium oxylobum Boiss. \& Buhse - Syn: G. leiocarpum Boiss. - En: Mountain Horned-poppy. Ar: Mamita Malsaa Al Thamar. - Lf: Annual herb, 30-40 cm. - Ft: April-August. - Mt Hermon: 2222 m, 33 25'44”N, 35 50'25'E. - Lebanon: Jabal El Barouk, Jabal El Knisse, Faraiya, Ariz, Baalbek, Zahle, Foum El Mizhab, Ouadi El Arayech. - Hab: Calcareous rocky soil mainly in high mountain ranges, sunny places. - Uses: Decoction of aerial parts is used to treat menstrual problems.

Papaver libanoticum Boiss. - En: Red Poppy, Field Poppy. - Ar: Khoshkhash Lebnan. - Lf: Annual herb, 15-30 cm. - Ft: May-July. - Mt Hermon: 1232 m, 33¹8'40”N, 35 51'12”E. - Lebanon: Jabal Sannine, Makmel, Ariz, Dahr Al Baidar, Qurnat as Sawda. Endemic to Lebanon and Syria. - Hab: Pastures, red gravel soil, low mountain ranges, sunny places. - Uses: Infusion of petals is used as emollient, expectorant, sudorific and sedative, for coughs, and the infusion of fruit capsules is given to babies as soporific.

Papaver minus (Bél.) Meikle - Syn: P. belangeri Boiss., P. argemone subsp. belangeri Takht., $P$. argemone subsp. minus (Bél.) Kadereit - En: Long Pricklyhead Melic Poppy, Pale Poppy. - Ar: Khoshkhash Arghemon. - Lf: Annual herb, 10-30 cm. - Ft: March-May. - Mt Hermon: 1250 m, $33^{\circ} 28^{\prime} 54^{\prime \prime}$, 35 51'35'E. - Lebanon: Bikfayia, Ain Zhalta, Afqa, Yammoune, Rayak, Ouadi El Harir. - Hab: Fields, woodlands, roadsides, gravel soil, sunny places. - Uses: None documented.

Papaver umbonatum Boiss. - Syn: Papaver syriacum Boiss. \& C. I. Blanche - En: Long-headed Poppy, Blindeyes. - Ar: Khoshkhash Mhayyar Aamlas. - Lf: Annual plant, 20-80 cm. - Ft: MarchJuly. - Mt Hermon: 1292 m, 3328'54”N, 35 51'35”E. Lebanon: Tyre, Beirut, Nahr El Kalb, Hammana, Tannourine, Ariz, Ehmej, Jabal El Barouk. - Hab: Meadows, fields, open shrubby areas, cultivated areas, roadsides, sunny places. - Uses: Medicinal plant, decoction of fruits is used to treat measles in children.

\section{Pinaceae}

Cedrus libani A. Rich. - Syn: C. libanensis Juss., C. libanotica Link, Pinus cedrus L. - En: Cedar of Lebanon. - Ar: Ariz Lebnani. - Lf: Evergreen coniferous tree, up to 40 m. - Ft: September-November. Mt Hermon: 1243 m, 33 $28^{\prime} 40^{\prime} \mathrm{N}, 35^{\circ} 51^{\prime} 41^{\prime \prime}$, naturalized. - Lebanon: Jabal Niha, Above Maaser El Shouf, Barouk, Ain Zhalta, Jabal El Jaj, Tannourine, Hadath, Bcharre, Ehden. - Hab: Mountain rocks, calcareous plateaus. - Uses: Powdered bark is externally applied to wounds as astringent.

\section{Plumbaginaceae}

Acantholimon antilibanoticum Mouterde - En: Anti-Lebanon Prickly-thrift. - Ar: Ghamloul Al Sharkyia. - Lf: Dwarf shrub, thorny cushion, 10-15 cm. - Ft: August-September. - Mt Hermon: 1843 m, 3326'24'N, 35 50'48'E. - Lebanon: Raifoun, Wadi Ibriza, Jabal El Homr, Marjhine, Tannourine. Endemic to Lebanon and Syria. - Hab: Mountain rocks, calcareous plateaus, red clay soil (Terra rossa) at medium and high altitudes. - Uses: Dried powdered roots are used for skin infections and wounds.

\section{Poaceae (Gramineae)}

Aegilops columnaris Zhuk. - En: Columnar Goat-grass. - Ar: Shaeer Amoudi. - Lf : Annual herb, 30-50 cm. - Ft: April-May. - Mt Hermon: 1191 m, 33²8’55’N, 3551'37’'E. - Lebanon: Jabal El 
Hadid, Qaa El Rim, Rachaiya. - Hab: Grassy grounds, pastures, scrubs, open shrubby places, roadsides, sunny areas. - Uses: None documented.

Aegilops neglecta Bertol. - Syn: A. ovata auct., A. triaristata Willd. - En: Ovate Goat-grass. - Ar: Shaeer Blees. - Lf: Annual herb, 10-60 cm. - Ft: March-June. - Mt Hermon: 1243 m, 33 28 '40”N, 3551'41”E. - Lebanon: Beirut, Hazmiyeh, Tripoli, Mkalles, Barouk, Bhamdoun, Mrouj, Terbol, Niha, Jabal El Knisse, Zahle, Rouissat, Hasroun, Tannayel, Ainata, Baalbek. - Hab: Dry and rocky slopes, scrubs, roadsides, fields, abandoned lands, sunny places. - Uses: Seeds are edible and used in the treatment of gout. The plant is recognized as a forage grass and fodder for goats and sheep.

Avena sterilis L. - En: Animated Oat, Sterile Oat, Wild Oat. - Ar: Shufan Barri, Ziwan. - Lf : Annual herb, up to 1 m. - Ft: March-May. - Mt Hermon: 1250 m, 33'28'54'N, 35'51'35'E. - Lebanon: Saida, Kahale, Beirut, Qalamoun, Jamhour, Aachqout, Damour, Bikfayia, Douma, Ras El Metn, Aabey, Dimane, Qannoubine, Hasbaya, Zahle, Chtaura. - Hab: Scrubs, meadows, cultivated lands, roadsides, dry and sunny places. - Uses: Decoction of flowering plant is used as diuretic and to treat arthritis, rheumatism, and skin diseases.

Elytrigia libanotica (Hack.) Holub - Syn: Agropyron libanoticum Hack., Elymus libanoticus (Hack.) Melderis - En: Lebanon Couch-grass. - Ar: Sifon Lebnani. - Lf: Perennial herb, 30-100 cm. - Ft: May-July. - Mt Hermon: 1243 m, 33²8’40”N, 3551’41’'E. - Lebanon: Jabal El Barouk, Akoura, Dahr Al Baidar, Ariz Bcharre, Ehmej, Ariz, Jabal Sannine, Qurnat as Sawda, Makmel, Yanta (Mt Hermon region). Endemic to Lebanon and Syria. - Hab: Scrubs, grazing pastures, red soil (Terra rossa), low mountain ranges. - Uses: Decoction of rhizomes is used as diuretic and to treat urinary disorders.

Melica cretica Boiss. \& Heldr. - Syn: M. ciliata var. laxiflora (Boiss. \& C. I. Blanche) Papp, M. ciliata var. villigera Bornm. - En: Hairy or Silky Melic. - Ar: Malika. - Lf: Perennial herb, 25-60 cm. - Ft: April-June. - Mt Hermon: 1250 m, 33²8'54'N, 3551'35”E. - Lebanon: Maasser El Chouf, Jabal El Barouk, Dahr Al Baidar, Knisse, Sannine, Ariz, Yammoune, Ain El Karn, Chtaura. - Hab: Calcareous plateaus, rocky slopes, scrubs, low mountain ranges, dry and sunny places. Uses: None documented.

Melica persica Kunth - Syn: M. cupani var. inaequiglumis Boiss. - En: Unequal-glumed Melic. Ar: Melika Motabayenat El Aasfat. - Lf: Perennial herb, 30-60 cm. - Ft: April-July. - Mt Hermon 1181 m, 35 28'41'N, 35 50'27'E. - Lebanon: Jabal El Barouk, Zahle, Baalbek, Jezzine, Ain Zhalta, Bchamoun, Douma, Hasroun, Tartij, Hasroun, Bcharre, Sannine, Qaa Er Rim, Ammiq, Ouadi El Harir, Yanta. - Hab: Among rubble and cracks in concrete, roadsides. - Uses: None documented.

Phalaris minor Retz. - En: Little Seed Canary Grass. - Ar: Shaeer El Far. - Lf: Annual herb. - Ft: March-June. - Mt Hermon: 1147 m, 3330’07’N, 3549’41”E. - Lebanon: Tyre, Beirut, Bouchrieh, Tripoli, Qaa. - Hab: Meadows, forests, pastures, roadsides, sunny places. - Uses: The plant is indicated as a hallucinogen and as a fodder for animals and birds. It may be toxic.

Triticum turgidum subsp. dicoccoides (Körn.) Thell. - Syn: T. dicoccoides (Körn.) Schweinf., T. sativum subsp. dicoccoides (Körn.) Asch. \& Graebn., T. vulgare var dicoccoides Körn. - En: Emmer Wheat. - Ar: Kamh Tounaai Al Habba. - Lf: Annual herb, 1 m. - Ft: May-July. - Mt Hermon: 1250 m, 3328'54'N, 3551'35'E. - Lebanon: Bouarij, Wadi Al Aarayech, Ouadi El Harir, Kfar Qouq, Rachaiya. - Hab: Cultivated lands, meadows, low mountain ranges, dry and sunny places. - Uses: Traditional food plant, seeds are edible. 


\section{Polygonaceae}

Polygonum cedrorum Boiss. \& Kotschy - En: Cedar Knotweed. - Ar: Asa El Arz. - Lf: Perennial plant, up to $50 \mathrm{~cm}$. - Ft: July-Nov. - Mt Hermon: 1213 m, 33²9'10'N, 35'51'26”'E. - Lebanon: Machghara, Jabal El Barouk, Jabal El Knisse, Dahr Al Baidar, Sannine, Hasroun, Laqlouq, Tannourine, Qamouaa, Yammoune, Zahle, Taanayel, Baalbek, Rachaiya. Endemic to Lebanon and Syria. - Hab: Mountain rocks, crevices, roadsides, dry and warm places. - Uses: Fresh leaves are edible. Decoction of aerial parts is used as a diuretic against kidney stones disease and in the treatment of diabetes and stomach diseases.

Rumex bucephalophorus L. subsp. bucephalophorus - Syn: R. bucephalophorus subsp. hipporegii (Steinh.) Rech. f. - En: Horned Dock. - Ar: Hommaid Ras El Tawr. - Lf: Annual herb, 30 cm. - Ft: February-May. - Mt Hermon: 1252 m, 33³0'01'N, 35 51'03'E. - Lebanon: Saida, Khalde, Bir Hassan, Beirut, Antilias, Nahr El Kalb, Mairouba, Raifoun, Bikfayia, Rachaiya. - Hab: Moist fields, among mountain rocks, woodlands, low mountain ranges, sunny places. - Uses: Fresh plant is edible. Decoction of aerial parts is orally used in treatment of jaundice and constipation.

\section{Primulaceae}

Cyclamen coum Mill. subsp. coum - En: Round-leaved Cyclamen. - Ar: Bakhour Mariam Jabali. - Lf: Perennial herb, tuberous geophyte, 10-20 cm. - Ft: February-April. - Mt Hermon: $1677 \mathrm{~m}$, $33^{\circ} 26^{\prime} 41^{\prime \prime N}, 35^{\circ} 50^{\prime} 32^{\prime \prime} E$. - Lebanon: Ghazir, El Kherbe, Qartaba, Faraiya, Chahtoul, Ariz El Barouk, Maasser El Chouf, Ariz, Dahr Al Baidar, Zahle, Ehden, Qamouaa forests, Jabal Foghri. Hab: Among the mountain rocks, on plant debris, red soil (Terra rossa), rock fissures, damp and sunny places. - Uses: Fresh plant is locally applied against ecchymosis and plant extract is believed to have antitumor properties. Pounded tubers mixed with powdered storax is used as ichthyotoxic.

\section{Ranunculaceae}

Adonis flammea L. - En: Scarlet Pheasant's-eye. - Ar: Adonees Ahmar. - Lf: Annual herb, 20-50 cm. - Ft: March-June. - Mt Hermon: 1239 m, 3328’38’N, 3551'12”'E. - Lebanon: Ehden, Ammiq, Deir Zenoun, Ouadi El Arayech, Ouadi El Harir. - Hab: Fields, pastures, roadsides, red gravel soil, sunny places. - Uses: Entire plant is used as emmenagogue, diuretic and in the treatment of gout and hydropsy. Also an ornamental plant.

Adonis microcarpa DC. - Syn: A. dentata subsp. microcarpa (DC.) Riedl, A. intermedia Webb \& Berthel., A. dentata subsp. intermedia (Webb \& Berthel.) Riedl, A. microcarpa subsp. intermedia (Webb \& Berthel.) Nyman, A. cupaniana auct. non Guss. - En: Small-fruit Pheasant's Eye; Red Chamomile. - Ar: Adonees Saghir Al Thamar. - Lf: Annual herb, 20-30 cm. - Ft: March-June. - Mt Hermon: 1182 m, 33²9'59’N, 3549'52'E. - Lebanon: Bir Hassan, Jezzine, Jabal Terbol, Nabatieh, Kalaat Chkif, Chtaura, Baalbek, Rachaiya. - Hab: Grassy and stony grounds, meadows, cultivated and abandoned lands, sunny places. - Uses: Decoction of whole plant is used as tonicardiac and diuretic in the treatment of hydropsy and gout.

Anemone coronaria L. - En: Poppy Anemone, Spanish Marigold. - Ar: Shakaek Numan. - Lf: Perennial herbaceous geophyte, 7-35 cm. - Ft: February-April. - Hab: Grassy grounds, vineyards, cultivated and abandoned lands, roadsides, sunny places.

var. alba (Goaty \&Pons) Burnat - Mt Hermon: $1168 \mathrm{~m}, 33^{\circ} 30^{\prime} 02^{\prime \prime} \mathrm{N}, 35^{\circ} 49^{\prime} 51^{\prime \prime} \mathrm{E}$.

var. phoenicea Ard. - Mt Hermon: 1140 m, 33³0'07'N, 35 49'41'E. - Lebanon: Saida, 
Kahale, Beirut, Nahr El Kalb, Tripoli, Aley, Mansouriyeh, Hasroun, Zahle, Deir Tamich, Taanayel, Bkirke. - Uses: Ornamental.

var. cyanea (Risso) Ardoino - Mt Hermon: 1140 m, 33 30'07'N, 35'49'41'"E. - Lebanon: Saida, Khalde, Ras Jedra, Kahale, Beirut, Nahr El Kalb, Tripoli, Beit Meri, Bsalim, Bhannes, Maamelteine, Harissa, Qartaba.

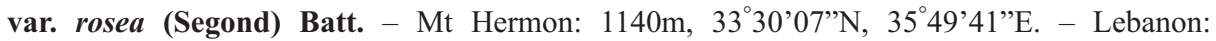
Kesrouane, Jounieh, Zouq Mkayel, Ghosta, Ghazir. - Uses: Medicinal, decoction of aerial parts is plant used to reduce hyperactivity, treatment of insomnia and irritability, cough, asthma and as a wash of skin ulcers and cleansing eye drop. Ornamental.

Clematis flammula L. - Syn: C. maritima L., C. flammula var. maritima (L.) DC. - En: Fragrant Virgin's Bower. - Ar: Zayyan. - Lf: Woody climber, up to $10 \mathrm{~m}$. - Ft: April-August. - Mt Hermon: 1300 m, 3328'55'N, 35 51'37'E. - Lebanon: Beirut, Nahr El Mout, Tripoli, Aabey, Jamhour, Deir El Qamar, Aamchit, Raifoun, Ehden, Wadi Qannoubine. - Hab: Woodlands, scrubs, hedges, sunny and shady places. - Uses: Plant decoction is internally used as hydragogue, diuretic, strong purgative for scabies and skin itching. The plant is also believed to be a cancer cure.

Delphinium peregrinum L. - Syn: D. junceum DC. - En: Violet Larkspur. - Ar: Rejl El Qonboura. - Lf: Perennial herb, 30-100 cm. - Ft: May-August. - Mt Hermon: 1290 m, 33 23'06”N, 35 51'18”'E. - Lebanon: Beirut, Nahr El Kalb, Tripoli, Aabey, Kfar Aaqab, Sawfar, Hrajel, Akoura, Faraiya, Hasroun, Ain Dara, Zahle, Ain Aata. - Hab: Scrubs, hedges, woodland and cultivated fields margins, generally on calcareous soil, roadsides. - Uses: Whole plant, particularly the young parts, is considered toxic to humans. Symptoms include skin irritation and severe digestive discomfort if ingested. It can cause cattle poisoning. Death may occur in a few hours of ingestion by cardiotoxic and neuromuscular block effects. The alcoholic solution of seeds is locally applied to the heads of children as a pediculicide. Ornamental.

Ficaria verna subsp. ficariiformis (F. W. Schultz) B. Walln. - Syn: F. grandiflora Robert, Ranunculus ficariiformis F. W. Schultz, R. ficaria subsp. ficariiformis (F. W. Schultz) Rouy \& Foucaud - En: Lesser Celandine. - Ar: Oshbet El Bawaseer. - Lf: Perennial herb, 10-40 cm. - Ft: January-April. - Mt Hermon: 1140 m, 3330’07’N, 3549'41'E. - Lebanon: Saida, Beirut, Jounieh, Tripoli, Qraiye, Ghazir, Zahle, Niha, Baalbek. - Hab: Rich clay soil, damp meadows, low mountain ranges, sunny places. - Uses: Entire plant is used as antidiarrhoeic and in the treatment of colon inflammation.

Ranunculus millefolius subsp. hiersolymitanus (Boiss.) P. H. Davis - Syn: R. hierosolymitanus Boiss., R. myriophyllus var. hierosolymitanus (Boiss.) Post - En: Jerusalem Buttercup. - Ar: Houzan El Qods. - Lf: Annual herb, 50-80 cm. - Ft: March-May. - Mt Hermon: 1140 m, 3330'07'N, 35'49'41'E. - Lebanon: Tripoli, Beirut, Jamhour, Broumana, Machnaka, Ras El Kifa, Aramoun, Jabal Terbol, Raifoun, Nabatieh, Mdeyrej, Dahr Al Baidar, Sannine, Bcharre, Hadath, Hadchit, Sawfar, Ehden, Hasroun, Ksara, Zahle, Nabee El Hadid, Ebel Es Saqi, Hasbani, Rachaiya and Yanta. - Hab: Meadows, pastures, calcareous rock crevices and hedges, roadsides, sunny places. - Uses: Pounded fresh flowers are externally used to treat rheumatism.

\section{Rhamnaceae}

Rhamnus punctata Boiss. - En: Thicket Rose. - Ar: Ward Al-Hayshah. - Lf: Deciduous shrub, 1-2 m. Mt Hermon: 1492 m, 3328'40’N, 35 50'27'E. - Lebanon: Saida, Nahr Ghadir, Choueifat, Beirut, Antilias, Nahr El Kalb, Tripoli, Nahr Abou Ali, Mar Roukhos, Kesrwan, Araya, Qraiye, Jamhour, 
Aintoura, Aley, Douma, Afqa, Ain Zhalta, Qannoubine, Ammiq, Deir Mimas, Rachaiya. - Hab: Meadows, roadsides, margins of cultivated fields, red clay soil (terra rossa). - Uses: None documented.

\section{Rosaceae}

Cotoneaster nummularius Fisch. \& C. A. Mey. - Syn: C. racemiflorus var. nummularius (Fisch. \& C. A. Mey.) Dippel - En: Nummular Cotoneaster. - Ar: Sfarjaleyyi. - Lf: Deciduous shrub, 50-150 cm. - Ft: May-June. - Mt Hermon: 2306 m, 33²5'37’N, 3550’32”E. - Lebanon: Mdeyrej, Jabal El Knisse, Kfar Selouane, Zahle, Afqa, Ariz, Hermel, Michmich, Ehden, Hadchit, Dimane, Bcharre, Ehden, Sir Al Danniyeh, Ainata, Jdita, Mt Hermon. - Hab: Calcareous plateaus, among rocks, high mountain ranges, dry and sunny places. - Uses: Decoction of fruits is taken orally as orexigenic, stomachic and expectorant.

Crataegus azarolus L. - Syn: C. aronia (L.) DC., C. azarolus subsp. aronia (L.) Rouy \& E. G. Camus - En: Mediterranean Medlar, Azarole, Hawthorn. - Ar: Zaarour Asfar. - Lf: Deciduous shrub or tree, 2-10 m. - Ft: March-May. - Mt Hermon: 1325 m, 33²8'27'N, 35 50'39'E. - Lebanon: Saida, Hadath, Beirut, Tripoli, Aley, Bsous, Jamhour, Hasroun, Souk El Gharb, Choueifat, Ain Zhalta, Ainata, Wadi El Harir. - Hab: Open shrubby areas, hedges. - Uses: Infusion of flowers is used as sedative and to treat chronic diarrhoea, insomnia and palpitation.

Potentilla geranioides subsp. syriaca (Boiss.) Soják - En: Syria Cinquefoil. - Ar: Mokaweya Soreyya. - Lf: Perennial herb with woody base, 10-30 cm. - Ft: April-July. - Mt Hermon: 2307 m, $33^{\circ} 25^{\prime} 39$ 'N, 35 50’31'E. - Lebanon: Jabal El Barouk, Jabal El Knisse, Makmel, Sannine, Ehden, Ariz, Jroud Tannourine, Ainata. Endemic to Lebanon and Syria. - Hab: Rich clay soil, damp meadows, low mountain ranges, sunny places. - Uses: Decoction of rhizomes is used as antidiarrhoeic, astringent, antipyretic, stomachic and depurative.

Prunus cocomilia Ten. - Syn: P. ursina Kotschy - En: Bear's Plum. - Ar: Brakrouk. - Lf: Deciduous shrub or tree, 4-8 m. - Ft: March-May. - Mt Hermon: 1191 m, 3328'55'N, 35 51'73"E. - Lebanon: Aramoun, Achkout, Qraiye, Beit Meri, Ehden, Sir Al Danniyeh, Bcharre, Barouk, Ain Zhalta, Dahr Al Baidar, Bouarij, Wadi El Harir, Rachaiya, Sawfar, Ainata. - Hab: Rocky habitats, roadsides, field boundaries and orchards. - Uses: Fruits are edible and used as laxative. Trunk and stems are used as fuel and in making agricultural tools.

Prunus dulcis (Mill.) D. A. Webb - Syn: P. korshinskyi Hand.-Mazz., Amygdalus korshinskyi (Hand.Mazz.) Bornm., A. communis L. var. microphylla Post - Ar: Lawz Korshinski. - Lf: Deciduous shrub or small tree, 1-2 m. - Ft: March-April. - Mt Hermon: 1550 m, 33 27'21'N, 35'52'39'E. - Lebanon: Jezzine, Machghara, Maasser El Chouf, Ain Zhalta Ariz, Zahle, Ammiq, Hazerta, Kefraiya, Ainata, Deir El Ahmar, Rachaiya. - Hab: Dry and rocky slopes, red gravel soil, medium mountain ranges, sunny places. - Uses: Decoction of seeds is taken orally to treat hypercholesterolemia.

Prunus prostrata Labill. - Syn: Cerasus prostrata (Labill.) Ser. - En: Mountain Cherry. - Ar: Karaz Barri. - Lf: Deciduous shrub, 20-80 cm. - Ft: April-June. - Mt Hermon: 1843 m, 3326'24'N, 35 50'48”E. - Lebanon: Hasroun, Jabal El Barouk, Ain Zhalta, Zahle, Ehden, Sannine, Jabal El Makmel, Mt Hermon, Ainata, Ain Hazir. - Hab: Calcareous open rocky slopes, flat clayey areas, high mountain ranges. - Uses: Ripe fruits are laxative.

Pyrus syriaca Boiss. - En: Syrian Pear. - Ar: Ngas Barri. - Lf: Deciduous shrub or tree, 5-10 m. Ft: February-May. - Mt Hermon: 1422 m, 33²7’22”N, 3550'50”E. - Lebanon: Saida, Beirut, 
Tripoli, Aabey, Baabda, Arayia, Jounieh, Ain Zhalta, Bcharre, Ariz, Dahr Al Baidar, Ainata, Hasroun, Deir El Ahmar, Baalbek, Deir El Achayer, Mt Hermon. - Hab: Rocky and cultivated lands, dry and sunny places. - Uses: Infusion of fruits is taken orally as expectorant to treat stomachache.

Rosa canina L. s. str. - En: Dog Rose. - Ar: Ward Barri. - Lf: Shrub, 1-2.5 m. - Ft: May-August. - Mt Hermon: 1292 m, 3328'55'N, 3551'37’E. - Lebanon: Raifoun, Baskinta, Ain Zhalta, Jabal Moussa, Ksara, Ouadi El Arayech, Baalbek, Ain Bourday, Rachaiya. - Hab: Hedges, among rocks, field margins, dry and damp places. - Uses: Fruit juice is used as astringent, antidiarrhoeic and antiscorbutic. Decoction of roots and fruits is taken to treat dyspnoea and rheumatism.

Rosa corymbifera Borkh. - Syn: $R$. dumetorum Thuill., $R$. canina var. collina Boiss. - En: Thicket Rose. - Ar: Ward Al-Hayshah. - Lf: Deciduous shrub, 1-2.5 m. - Ft: May-June. - Mt Hermon: 1220

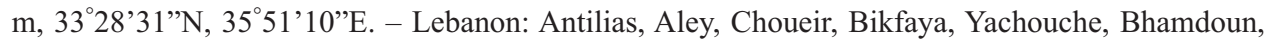
Sawfar, Hasroun, Ehden, Ariz, Ainata, Zahle, Baalbek. - Hab: Meadows, roadsides, margins of cultivated fields, red clay soil (terra rossa). - Uses: Ornemental plant.

Rosa pulverulenta M. Bieb. - Syn: R. glutinosa Sm., R. libanotica Boiss. - En: Sticky Rose. - Ar: Ward Dabek. - Lf: Deciduous shrub, 20-50 cm. - Ft: June-July. - Mt Hermon: 1289 m, 33²6'33”N, 35 51'15'E. - Lebanon: Jabal El Barouk, Jabal El Knisse, Jabal Sannine, Dimane, Ariz, Ehden, Yammoune, Jabal Qamouaa, Qalaat Arouba, Mt Hermon. - Hab: Hedges, among rocks, field margins, several mountain ranges, dry and damp places. - Uses: Fruit juice is orally used as astringent, antidiarrhoeic, diuretic and antiscorbutic.

Rubus canescens DC. - Syn: R. tomentosus auct. sensu Weihe \& Nees - En: Tomentose blackberry. - Ar: Olaik Abyad. - Lf: Deciduous shrub, 80 cm, June-October - Mt Hermon: 1197 m, 33²9'47'N, 3550’35’E. - Lebanon: Raifoun, Douma, Ghosta, Kfar Selouane, Jabal El Knisse, Jabal El Barouk, Dimane, Sannine, Zahle, Hezerta, Mt Hermon. - Hab: Thickets, hedges, fields, rocky limestone habitats. - Uses: Fruits are edible and decoction of leaves is used as astringent.

Sanguisorba minor subsp. lasiocarpa (Boiss. \& Hausskn.) Nordborg - Syn: Poterium compactum Boiss. - En: Compact burnet. - Ar: Ballan Katheef. - Lf: Perennial, rhizomatous herb. - Ft: JuneJuly. - Mt Hermon : 1140 m, 33³0'07’N, 3549'41'E. - Lebanon: between Jabal Sannine and Jabal El Knisse, North Baskinta. - Hab: Among rocks, gravel soil, roadsides, sunny places. - Uses: Decoction of the entire plant (roots and leaves) is used as astringent and vulnerary to cure wounds. Leaves infusion is used as a soothing treatment for sunburn and eczema.

Sanguisorba verrucosa (G. Don) Ces. - Syn: Poterium verrucosum G. Don - En: Warty Burnet. - Ar: Ballan Taloul. - Lf: Perennial, rhizomatous herb, 20-50 cm. - Ft: April-May. - Mt Hermon: 1405 m, 33 27’42”N, 35 50’35”E. - Lebanon: Saida, Beirut, Ras Chekka, Tripoli, Nahr Abou Ali, Beit Meri, Jamhour, Jabal Terbol, Hadchit, Ehden, Qannoubine, Yammoune, Zahle, Qoub Elias, Rayak. - Hab: Meadows, grassy grounds, moist fields, roadsides, low mountain ranges, sunny places. - Uses: None documented.

Sarcopoterium spinosum (L.) Spach - Syn: Poterium spinosum L., Sanguisorba spinosa (L.) Bertol. - En: Spiny Burnet. - Ar: Ballan Shaek. - Lf: Dense, spiny dwarf shrub, $60 \mathrm{~cm}$. - Ft: March-April. - Mt Hermon: 1292 m, 3328'55”N, 3551'73”'E. - Lebanon: Widespread especially in overgrazed pastures. - Hab: Dry and rocky slopes, open woodlands, grassy grounds, sunny places. - Uses: Whole plant is used as firewood for villagers bread ovens "Tanour" for baking 
Lebanese bread "Markouk". Decoction of dried fleshy berries and root barks are orally used by Bedouin healers and villagers to treat diabetes mellitus.

\section{Rubiaceae}

Crucianella macrostachya Boiss. - En: Common Crossword. - Ar: Solaybeyya Sagheerat El Sanabel. - Lf: Annual plant, 30-60 cm. - Ft: May-August. - Mt Hermon: 1325 m, 33²8'29'N, 35 50'37'E. - Lebanon: Saida, Beirut, Marjaayoun, Nahr El Mout, Tripoli, Bikfayia, Bhamdoun, Kahale, Jabal El Barouk, Chebaa, Akkar, Rachaiya. - Hab: Rocky slopes, gravel, abandoned lands. - Uses: No indication.

Galium divaricatum Lam. - En: Lamarck's bedstraw. - Ar: Galium Rafee. - Lf: Annual herb, 1040 cm. - Ft: March-April. - Mt Hermon: 1140 m, 33 30’07'N, 3549'41”E. - Lebanon: Beirut, Beit Meri, Baabda, Hammana, Mairouba, Faraiya, Hammara. - Hab: rocky slopes, shaded regions, noncalcareous soils, low mountain ranges. - Uses: Medicinal plant, decoction of whole plant is used as diuretic and vulnerary.

Galium incanum subsp. antilibanoticum Ehrend. - Syn: G. orientale var. alpinum Boiss. sensu Bornm. in sched. - Ar: Galium Mbayyad. - Lf: Perennial herb, 15-30 cm. - Ft: May-June. - Mt Hermon: 2072 m, 33²5’58’N, 3550'20”E. - Lebanon: Bcharre, Kalat Djendal (Mt Hermon region, locus classicus). Endemic to Lebanon and Syria (see map in Ehrendorfer 1951: 446). - Hab: Cliffs of dry stony slopes in high mountain ranges. - Uses: None documented.

Galium libanoticum Ehrend. - Syn: G. scabrifolium (Boiss.) Hausskn. f. libanotica Bornm. in sched. - En: Lebanon Bedstraw. - Ar: Ghalium Lebnan. - Lf: Perennial herb, 30-60 cm. - Ft: JuneJuly. - Mt Hermon: 2057 m, 3326’01'N, 35 50’21'E. - Lebanon: Mairouba, Bchela, Bzoummar, Raifoun, Afqa, Bikfayia, Barouk, Mdeyrej, Aaquora, Ehmej, Bcharre, Dimane, Hasroun, Zahle, Kfar Selouane, Jabal Sannine, Ammiq, Baalbek, Yanta, Ehden, Ouadi El Arayech, Hasbaya and Rachaiya. - Hab: Dry and calcareous rocky slopes, sunny places at medium and high altitudes. - Uses: Decoction of entire plant is used as diuretic and vulnerary and for wounds.

Mericarpaea ciliata (Banks \& Sol.) Eig - Syn: Mericarpaea vaillantioides Boiss. - En: False Valantia. - Ar: Shabeehat El Valantia. - Lf: Annual herb, 5-15 cm. - Ft: March-April. - Mt Hermon: 1182 m, 3329'59”N, 3549'52”E. - Lebanon: Al Masnaa, Deir El Aachayer, Ouadi El Harir, Rachaiya. - Hab: Meadows, red soil (Terra Rossa), dry and sunny places. - Uses: None documented.

Rubia tenuifolia subsp. doniettii (Griseb.) Ehrend. \& Schönb.-Tem. - Syn: R. doniettii Griseb., $R$. tenuifolia d'Urv. var. stenophylla (Boiss.) J. Thiébaut, $R$. olivieri A. Rich. var. stenophylla (Boiss.) Boiss. - En: Elliptic-leaved Madder. - Ar: Fowwa Dayyekat El Warak. - Lf: Perennial climber with woody base, 30-120 cm. - Ft: March-June. - Mt Hermon: 1355 m, 33 28'29'N, 35 50'37'E. Lebanon: Beirut, Aabey, Terbol, Bikfaiya, Aley, Kahale, Afqa, Hasroun, Sannine, Ariz, Zahle, Baalbek, Ouadi El Harir, Rachaiya and Chebaa. - Hab: Mountain rocks, woodlands, scrubs, thickets, cultivated land margins, low mountain ranges, sunny places. - Uses: Decoction of root is used as diuretic, laxative, emmenagogue, and abortive. The mixture of roots decoction and oil is locally applied to treat sciatica and rheumatism, infusion of flowers is used as antidiarrhoeic and aphrodisiac. 
Scrophulariaceae s. 1. (incl. Plantaginaceae p. pte.)

Anarrhinum orientale Benth. - En: Oriental Anarrhium. - Ar: Sawsal Sharki. - Lf: Perennial herb, 30-80 cm. - Ft: May-July. - Mt Hermon: 1355 m, 33²8'29'N, 35'50'37'E. - Lebanon: Saida, Beirut, Nahr El Kalb, Deir El Qamar, Bikfayia, Ghazir, Baabda, Aintoura, Chouiefat, Mokhtara, Mansouriye, Maasser El Chouf, Faraiya, Douma, Ehden, Ehmej, Bcharre, Zahle, Baalbek, Yanta and Rachaiya. - Hab: Scrubs, meadows, rocky slopes, roadsides, dry and sunny places. - Uses: None documented.

Scrophularia libanotica subsp. libanotica var. australis R. R. Mill - Syn: S. variegata Bieb. var. libanotica Boiss. - En: Lebanon Fig-wort. - Ar: Khanzireyyi Lebnan. - Lf: Perennial herb, 20-50 cm. Ft: May-July. - Mt Hermon: 1923 m, 33²6'17'N, 3550'37'E. - Lebanon: Qornet el Aachra, Qurnat as Sawda, Ouyoun Orghosh. - Hab: Dry scrubs, cultivated and abandoned lands, open shrubby areas, sunny places, roadsides at low and medium altitudes. - Uses: Infusion of flowering tops, leaves and rhizomes for $20 \mathrm{~min}$ is locally applied for the treatment of skin irritations, eczemas and wounds.

Verbascum agrimoniifolium (K. Koch) Hub.-Mor. - Syn: Celsia agrimoniaefolia K. Koch - En: Syrian Mullein. - Ar: Bosayr Souri. - Lf: Biennial rosette herb, 50-150 cm. - Ft: April-Oct. - Mt Hermon: 2100 m, 33 25’37’N, 35 50’32”E. - Lebanon: Tripoli, Furn El Chebbak, Jabal Sannine, Sawfar, Aitanit, Ksara, Ferzol, Zahle, Marj, Rachaiya. Endemic to Lebanon and Syria. - Hab: Hedges, roadsides, grassy and stony grounds, dry and sunny places. - Uses: Medicinal plant, decoction of flowers isused as emollient and diuretic and in the treatment of cough, bronchitis, cold, fever, diarrhea and gastrointestinal bleeding.

Verbascum cedreti Boiss. - Syn: V. rascheyanum Boiss., V. cedretioides Gombault - En: Cedar mullein. - Ar: Bosayr El Ariz. - Lf: Biennial herb, 30-80 cm. - Ft: May-August. - Mt Hermon: 1310 m, 33 29'02”N, 35 50'25’E. - Lebanon: Bikfaiya, Choueir, Machmouche near Jezzine, Maaser El Chouf, Kfar Halda near Douma, Tripoli, Fnaidek, Jabal El Barouk, Jabal El Knisse, Ain Zhalta, Jabal Sannine, Jisr Al Hajar, Aitou, Basloukit, Ehden, Bcharre, Ariz, Tannourine, Dimane, Dahrn El Kadib, Rachaiya. - Hab: Stony slopes, among mountain rocks, low mountain ranges, dry and sunny places. - Uses: Decoction of roots and leaves is used as emollient, anti-inflammatory and anti-poison.

Verbascum libanoticum Murb. \& J. Thiébaut - En: Lebanon Mullein. - Ar: Bosayr Lebnani. - Lf: Biennial herb, 50-120 cm. - Ft: May-July. - Mt Hermon: 1923 m, 33²6'17'N, 35 50'37'E. Lebanon: Qartaba, Chahtoul, Nahr Ed Dahab, Ehmej, Dhour Choueir. Endemic to Lebanon and Syria. - Hab: Calcareous rock-crevices, open dry shrubby areas, roadsides, sunny places. - Uses: Decoction of flowers and leaves emollient. Decoction in milk or water is locally applied on skin ulcers. Decoction of leaves mixed with Malva neglecta is used as poultice for hemorrhoids.

Verbascum ptychophyllum Boiss. - En: Wavy-leaved Mullein. - Ar: Bosayr Motamawej El Warak. - Lf: Biennial or perennial herb, 50-200 cm. - Ft: May-July. - Mt Hermon: 1191 m, 33 28'55'N, 35 51'73”'E. - Lebanon: Zahle, Ouadi El Harir, Jdeidit Yabous, Rachaiya. Endemic to Lebanon and Syria. - Hab: Calcareous plateaus, dry and stony hillslopes, sunny places. - Uses: Medicinal plant, decoction of leaves and flowers is used as emollient. Decoction of roots and leaves is used for the treatment of eye diseases and improving eye sight.

Verbascum sinaiticum Benth. - Syn: V. ternacha Hochst., V. somaliense Baker - En: Sinai Mullein. - Ar: Bosayr Seenaa. - Lf: Biennial herb, 60-150 cm. - Ft: June-August. - Mt Hermon: 1292 m, $33^{\circ} 28^{\prime} 55^{\prime}$ 'N, $35^{\circ} 51^{\prime} 73^{\prime \prime} E$. - Lebanon: Beit Chama, Ablah, Rayak, Yammoune, Rachaiya. - Hab: Dry 
and rocky slopes, gravel soil, roadsides, fields, low mountain ranges, sunny places. - Uses: Medicinal plant, flower and leaf are used as sedative, diuretic, emollient, astringent and in the treatment of cough and asthma. Essential oil of flowers is used for eczema, earache, catarrhs, and the treatment of warts and carbuncles.

Veronica syriaca Roem. \& Schult. - Syn: V. pedunculata Labill. non Vahl - En: Syrian Speedwell. - Ar: Veronika Soureyya. - Lf: Annual herb, 10-20 cm. - Ft: January-May. - Mt Hermon: 1182 m, 33 30'02”N, 35 49'51'E. - Lebanon: Saida, Beirut, Choueifat, Tripoli, Bikfaiya, Aley, Aabey, Raifoun, Chemlane, Jezzine, Aintoura, Mairouba, Bzemmar, Faraiya, Ehden, Ariz, Hasroun, Dahr Al Baidar, Bcharre, Sannine, Rachaiya. - Hab: Stony and rocky slopes, red gravel soil, roadsides, gardens, low mountain ranges. - Uses: None documented.

\section{Solanaceae}

Hyoscyamus albus L. - En: White Henbane. - Ar: Banj Aabyad. - Lf: Annual plant, 50-60 cm. - Ft: March-July. - Mt Hermon: 1229 m, 3329'08'N, 3551'29'E. - Lebanon: Enfe, Tripoli, Broumana, Amchit, Qraiye, Bchela, Harajel, Terbol, Salima, Baskinta, Machghara, Nabatieh, Rachaiya. - Hab: Old walls, roadsides, low mountain ranges, sunny places. - Uses: Hallucinogenic and poisonous plant.

Hyoscyamus reticulatus L. - En: Netted Henbane. - Ar: Banj Shabaki. - Lf: Annual herb, 2050 cm. - Ft: March-May. - Mt Hermon: 1224 m, 3329'08'N, 3551'29”'E. Lebanon:Yammoune, Ksara, Zahle, Marj, Majdal Anjar, Baalbek, Ain Bourday, Al Masnaa, Jabal El Barouk, Rachaiya. - Hab: Margins of cultivated fields and gardens, roadsides. - Uses : Hallucinogenic drug and poisonous plant.

\section{Valerianaceae}

Centranthus longiflorus Steven subsp. longiflorus - En: Long-flowered Spur-Valerian. - Ar: Asa El Natoor. - Lf: Annual herb, 60-150 cm. - Ft: June-Oct. - Mt Hermon: 1147 m, 33 30'07'N, 35 49'41'E. - Lebanon: Hammana, Bikfayia, Qraiye, Jabal El Barouk, Sawfar, Ehden, Hasroun, Ariz, Bcharre, Qannoubine, Ain Zhalta, Sannine, Ain Hazir, Zahle, Jdita, Ain Bourday, Baalbek, Mt Hermon. - Hab: Stony ground, roadsides, old walls, sunny places. - Uses: Medicinal and ornamental plant indicated as antitumour, anti-inflammatory, sedative and anticonvulsant.

\section{Discussion and Conclusions}

Our surveys launched in 2011 revealed the existence of an important flora on the Lebanese side of Mt Hermon. The first annotated checklist presented here counts a number of 223 species, around 110 of which were previously recorded as present in the investigated area (Mouterde 1966, 1970, 1983) and the remaining species (113) are newly reported. The families of Asteraceae (27 spp.), Lamiaceae (20 spp.) and Rosaceae (13 spp.) constituting the major share of the species form $40 \%$ of the list. From the reported 223 taxa, 24 are narrow endemics. This pronounced endemism represents the most important feature characterizing the flora of the mountain. The endemics are rather diffused over the different sites studied. These preliminary findings indicate the pronounced plant biodiversity richness of the Lebanese side of Mt Hermon. The climatic and geomorphological 
diversity and the isolation effect of its topography have rendered the mountain a home for a rich variety of wildlife including many rare and endemic plant species.

Although not yet completed, this work is the first comprehensive contribution to the checklist of the Hermon flora in Lebanon and can be considered the basis for compiling a complete checklist, and supporting the evaluation efforts of the diversity of the Lebanese flora in general. This study highlights the vital role Mt Hermon plays in the conservation of many economically important wild plants and in the resilience of local livelihoods.

On the other hand the gathered ethnobotanical knowledge reveals the importance of the mountain for the surrounding villages that have always benefited of the mountain plants as a major source for their primary needs (food, feed, medicines, energy, etc.). Indeed, the rich biodiverse environment of Mt Hermon has been providing a veritable trove of flora containing compounds of high value for the indigenous people. Although this traditional knowledge is inherited from generation to generation over hundreds of years and may not be based on scientific evidence, it appears to be efficient in treatment of many health disorders (Apostolides-Arnold 1991). Such knowledge stands as a potent source to derive inspiration towards biodiversity conservation while enhancing livelihoods and adapting to perturbations. It should be further enlarged to cover the whole flora of the mountain before being submitted to valorization, sustainable utilisation and capitalization. The people of the mountain should be trained on suitable cultivation, sustainable harvesting and post harvesting technology. They should be also encouraged to take up enterpreneurial actions to convert the bioresources of the mountain into socioeconomic wealth.

Therefore, it is strongly recommended to develop and implement specific regulations, monitoring systems and public awareness tools on preservation and sustainable use of the Lebanese flora. Moreover, scientific monitoring and accurate prediction of the future impacts of climate change on plant diversity are critical to the development of conservation strategies.

\section{References}

Abou Chaar, C. I. 2004: Medicinal Plants of Lebanon. - Archaeol. Hist. Lebanon 19: 70-85.

Abou Zakhem, A. 1989: A contribution to the identification of natural vegetation cover of Hermon Mountain. (In Arabic). - Damascus Univ. J. Agric. Sci. 17: 65-101.

Angiosperm Phylogeny Group [APG] 2009: An update of the Angiosperm Phylogeny Group classification for the orders and families of flowering plants: APG III. - Bot. J. Linn. Soc. 161: 105-121. doi: 10.1111/j.1095-8339.2009.00996.x

Arnold-Apostolides, N. 1991: Ethnobotanique et Ethnopharmacologie de la flore de Chypre et de l' Est méditerranéen. - Publ. Centre Rég. Phytosoc., 1-6. - Bailleul.

- 2004: Les plantes allergisantes de la flore sauvage ou cultivée du Liban. - Premier Congrès médical de la Société d'allergologie du Liban. Conférence et Publication Mai 2004, Beyrouth.

Auerbach, M. \& Shmida, A. 1993: Vegetation change along an altitudinal gradient on Mt. Hermon, Israel. - No evidence for discrete communities. - J. Ecol. 81: 25-33.

CEPF (Critical Ecosystem Partnership Fund by Birdlife International, LPO and DOPPS) 2010: Mediterranean Basin Biodiversity Hotspot - Ecosystem Profile.

Danin, A. 1992: Flora and vegetation of Israel and adjacent areas. - Bocconea 3: 18-42.

Davis, P. H. 1965-1985: Flora of Turkey and the East Aegean Islands, 1-9. - Edinburgh. 
Devesa J. A. \& López G. 1997: Nótas taxonómicas y nomenclaturales sobre el género Ononis (Leguminosae) en la Península Ibérica e Islas Baleares. - Anales Jard. Bot. Madrid 55: 245-260.

Ehrendorfer, F. 1951: Rassengliederung, Variabilitätszentren und geographische Merkmalsprogressionen als Ausdruck der raum-zeitlichen Entfaltung des Formenkreises Galium incanum S.S. - Oesterr. Bot. Z. 98: 427-490.

El Beyrouthy, M., Arnold, N., Delelis-Dusollier, A. \& Dupont, F. 2008: Plants used as remedies antirheumatic and antineuralgic in the traditional medicine of Lebanon. - J. Ethnopharmacol. 120: 315-334. doi: 10.1016/j.jep.2008.08.024

El Chaar, S. \& Arnold, N. 2005: Etude ethnobotanique et ethnopharmacologique des espèces médicinales sur le système broncho-pulmonaire . - Ann. Rech. Sci. Publ. Holy Spirit Univ. Kaslik, Fac. Sci. Agron. Aliment. 6: 145-176.

Euro+Med 2006-: Euro+Med PlantBase - the information resource for Euro-Mediterranean plant diversity. - Published on the Internet http://ww2.bgbm.org/EuroPlusMed/ [accessed 22 Dec. 2014].

Förther, H. \& Podlech, D. 1991: Revision der Ononis natrix-Gruppe (Leguminosae) von Makaronesien, Nordafrika und dem angrenzenden Westasien. - Mitt. Bot. Staatssamml. München 30: 197-296.

Fuentes-Bazan, S., Uotila, P. \& Borsch, T. 2012: A novel phylogeny-based generic classification for Chenopodium sensu lato, and a tribal rearrangement of Chenopodioideae (Chenopodiaceae). - Willdenowia 42: 5-24. doi: 10.3372/wi.42.4210

Gelderen, D. M. van, Jong, P. C. de \& Oterdoom, H. J. 1994: Maples of the world. - Portland, Oregon.

Greuter, W. (ed.) 2008: Med-Checklist. A critical inventory of vascular plants of the circum-mediterranean countries, 2. - Palermo, Genève \& Berlin.

—, Burdet, H. M. \& Long, G. 1984, 1986, 1989: Med-Checklist. A critical inventory of vascular plants of the circum-mediterranean countries, 1, 3, 4. - Genève, Berlin.

Maylychef, P. 1989: Les plantes médicinales au Liban. - The Lebanese Med. J. 38: 59-60.

Médail, F. \& Quézel, P. 1977: Hot-spots analysis for conservation of plant biodiversity in the Mediteranean Basin. - Ann. Missouri Bot. Gard. 84: 112-127.

— \& - 1999: Biodiversity hot-spots in the Mediterranean Basin: Setting global conservation priorities. - Conserv. Biol. 13(6): 1510-1513.

Ministry of Environment (MoE), GEF and UNDP 2009: Fourth National Report of Lebanon to the Convention on Biological Diversity, July 2009.

Ministry of Environment (MoE) - Lebanon, UNDP and Ecodit 2011: Environment in Lebanon; Status Report 2010.

Mouterde, P. 1966, 1970, 1983: Nouvelle Flore du Liban et de la Syrie, 1-3. - Beyrouth.

Nehmé, M. 2000: Etymological Dictionary of Lebanese Flora. Scientific Names and their Etymology. Arabic, English and French Names. - Beyrouth.

- 2008: Etymological Dictionary of Syrian Flora. Scientific Names and their Etymology. Arabic, English and French Names. - Beyrouth.

Podlech, D. \& Zarre, S. 2013: A taxonomic revision of the genus Astragalus L. (Leguminosae) in the Old World, 1-3. - Wien.

Saouma, A. \& Arnold N. A. 1991: Etude botanique et phytogéographique des plantes médicinales du Liban à effet diurétique, antilithiasique, lithontriptique et antispasmodique. - Publ. Holy Spirit Univ. Kaslik, Fac. Sci. Agron. Aliment. 11: 1-100.

Zohary, M. 1966, 1972, 1978, 1986: Flora Palaestina. Text, 1-4. - Jerusalem.

Yazbek, M., Machaka-Houri, N., Al-Zein, M. S., Safi, S., Sinno, N. \& Talhouk, S. N. T. 2011: Lebanon. - P. 53 in: Radford, E. A., Catullo, G. \& Montmollin, B. de (ed.), Important Plant 
Areas of the south and east Mediterranean region: Priority sites for conservation. - Gland, Switzerland \& Malaga.

Addresses of authors:

Nelly Arnold ${ }^{1 *}$, Safa Baydoun ${ }^{2 *+}$, Lamis Chalak $^{3} \&$ Thomas Raus ${ }^{4}$,

${ }^{1}$ Research Center for Environment and Development, Beirut Arab University, Lebanon, nellyarnol28@,gmail.com

${ }^{2}$ Safa Baydoun ${ }^{+}$, Research Center for Environment and Development, Beirut Arab University, Lebanon. P.O. Box: 115020 Beirut, Tel: +961 8 542051/ 543051, Fax: +961 8 544051, safaa.baydoun@bau.edu.lb

${ }^{3}$ Faculty of Agricultural Engineering \& Veterinary Medicine, Lebanese University, Dekwane, Beirut, Lebanon, lamis.chalak@gmail.com

${ }^{4}$ Botanischer Garten und Botanisches Museum Berlin-Dahlem, Freie Universität Berlin, Germany, t.raus@bgbm.org

*First and second authors have contributed equally to this manuscript.

${ }^{+}$Corresponding Author 
